\title{
Loss of glutathione redox homeostasis impairs proteostasis by inhibiting autophagy-dependent protein degradation
}

\author{
David Guerrero-Gómez ${ }^{1}$ José Antonio Mora-Lorca ${ }^{1,2}$ • Beatriz Sáenz-Narciso ${ }^{3}$ • Francisco José Naranjo-Galindo ${ }^{1}$. \\ Fernando Muñoz-Lobato ${ }^{1}$. Cristina Parrado-Fernández ${ }^{4}$ - Julen Goikolea ${ }^{4}$ - Ángel Cedazo-Minguez ${ }^{4}$. \\ Christopher D. Link ${ }^{5}$ Christian Neri ${ }^{6}$ María Dolores Sequedo ${ }^{7,8} \cdot$ Rafael P. Vázquez-Manrique $^{7,8}$. \\ Elena Fernández-Suárez ${ }^{9}$ - Veit Goder ${ }^{9} \cdot$ Roser Pané $^{10}$ • Elisa Cabiscol ${ }^{10}$ • Peter Askjaer ${ }^{11}$ - Juan Cabello ${ }^{3}$. \\ Antonio Miranda-Vizuete ${ }^{1}{ }^{1}$
}

Received: 26 June 2018 / Revised: 4 December 2018 / Accepted: 20 December 2018 / Published online: 15 February 2019

(c) ADMC Associazione Differenziamento e Morte Cellulare 2019

\begin{abstract}
In the presence of aggregation-prone proteins, the cytosol and endoplasmic reticulum (ER) undergo a dramatic shift in their respective redox status, with the cytosol becoming more oxidized and the ER more reducing. However, whether and how changes in the cellular redox status may affect protein aggregation is unknown. Here, we show that $C$. elegans loss-offunction mutants for the glutathione reductase $g s r-1$ gene enhance the deleterious phenotypes of heterologous human, as well as endogenous worm aggregation-prone proteins. These effects are phenocopied by the GSH-depleting agent diethyl maleate. Additionally, $g s r-1$ mutants abolish the nuclear translocation of HLH-30/TFEB transcription factor, a key inducer of autophagy, and strongly impair the degradation of the autophagy substrate p62/SQST-1::GFP, revealing glutathione reductase may have a role in the clearance of protein aggregates by autophagy. Blocking autophagy in gsr-l worms expressing aggregation-prone proteins results in strong synthetic developmental phenotypes and lethality, supporting the physiological importance of glutathione reductase in the regulation of misfolded protein clearance. Furthermore, impairing redox homeostasis in both yeast and mammalian cells induces toxicity phenotypes associated with protein aggregation. Together, our data reveal that glutathione redox homeostasis may be central to proteostasis maintenance through autophagy regulation.
\end{abstract}

\section{Introduction}

The maintenance of a functional proteome (also known as proteostasis or protein homeostasis) requires the concerted intervention of different enzymatic and regulatory systems,

These authors contributed equally: Juan Cabello, Antonio MirandaVizuete

Edited by H-U Simon

Supplementary information The online version of this article (https:// doi.org/10.1038/s41418-018-0270-9) contains supplementary material, which is available to authorized users.

Juan Cabello

juan.cabello@riojasalud.es

$\triangle$ Antonio Miranda-Vizuete amiranda-ibis@us.es

Extended author information available on the last page of the article termed as Proteostasis Network (PN). Thus, PN assists polypeptides to achieve and maintain their active conformation (chaperones and folding factors), provides an adequate cellular environment for their function (unfolded protein response (UPR), heat-shock, and oxidative responses) and efficiently disposes them when recognized as non-functional or are no longer required (proteasome and autophagy systems) [1, 2]. Given its pivotal role in cellular function, deregulation of PN dramatically impacts protein homeostasis and is an underlying cause of several human diseases, collectively known as proteinopathies, mainly characterized by the aberrant deposition of aggregated, misfolded proteins [3]. These disorders include some of the most prevalent neurodegenerative diseases like Alzheimer's, Parkinson's, or Huntington's diseases, among others [4]. At the core of PN, the pathways involved in reduction, oxidation, and isomerization of disulfide bonds are essential to maintain protein homeostasis as they prevent, correct, or remove faulty bonds leading to non-functional protein folding. 
Protein disulfide bonds are formed at cysteine residues, which can also undergo a variety of other post-translational modifications such as sulfenylation, sulfinylation, nitrosylation, or persulfidation, some of which are irreversible [5]. The maintenance of protein thiols in their reduced state in the cytosol and mitochondrial matrix is carried out mainly by two dedicated redox pathways: the thioredoxin and glutathione systems [6]. In contrast, in the lumen of the endoplasmic reticulum (ER) (the subcellular compartment where the folding of the proteins entering the secretory pathway takes place), the correct disulfide bond formation is performed by an oxidative folding pathway, largely composed of members of the protein disulfide isomerase (PDI) family [7]. Intriguingly, no members of the thioredoxin or glutathione redox systems exist in the ER, and the identity of the enzymatic systems providing reducing equivalents to ER-resident PDIs has remained elusive for decades. Recently, the cytoplasmic thioredoxin system has been shown to shuttle electrons into the ER to reduce oxidized PDIs and ensure correct disulfide formation [8]. In addition, an active import of cytoplasmic glutathione into ER lumen by specific transporters is key to maintain redox homeostasis in the ER [9], highlighting the importance of a coordinated redox network for maintaining proteostasis across all subcellular compartments.

Organisms as distant as the nematode Caenorhabditis elegans and humans contain a similar number of cysteine residues in their proteome, estimated in about 210,000 [10]. Importantly, their respective thioredoxin and glutathione redox systems are highly conserved [11], supporting the use of C. elegans as a simple model relevant to addressing key questions in redox biology [12, 13]. In worms, the thioredoxin system is dispensable for redox homeostasis as null or strong loss-of-function mutants of the genes encoding the cytoplasmic and mitochondrial thioredoxins (trx-1, trx-2, and $\operatorname{tr} x-3)$ and thioredoxin reductases (trxr-1 and trxr-2) are viable and superficially wild-type [14-17]. In contrast, mice lacking any member of the cytosolic or mitochondrial thioredoxin system are embryonic lethal (reviewed in [18]). However, when bypassing the embryonic requirement by employing conditional knock-outs, the thioredoxin system is also dispensable for viability in mice [18]. Thus, in the absence of a functional thioredoxin system, the glutathione system may be responsible for the maintenance of redox homeostasis in both organisms [16, 18, 19]. This key role of glutathione is further emphasized by the strict requirement of dietary methionine as a reduced glutathione (GSH) source (generated by the trans-sulfuration pathway) in the absence of functional thioredoxin and glutathione redox systems in mice [19] or the lethal phenotypes of mutations in the genes responsible for glutathione synthesis ( $g$ cs- 1 and gss$1)$ or recycling $(g s r-1)$ in worms $[10,20]$. Furthermore, a study in C. elegans showed that GSH redox potential is highly sensitive to small changes in its oxidation status [10]. This sensitivity would translate into rapid responses to even minor perturbations of the cytosolic redox environment and, as consequence, to a quick adjustment of the thiol-disulfide balance of the proteome. Together, these data strongly suggest that the glutathione system is the primary enzymatic system responsible for the maintenance of redox homeostasis in metazoa.

Under non-stressed conditions, the cytosol of eukaryotic cells is maintained in a reduced state to favor the stabilization of free thiol groups while the ER environment is more oxidized in order to promote disulfide bond formation [21]. Consistent with a tightly controlled interplay between proteostasis and redox homeostasis, proteotoxic stress generated by aggregating proteins in the cytoplasm of both C. elegans and mammalian cells strongly disturb redox homeostasis, causing a shift towards a more oxidizing condition in the cytosol and, conversely, towards a more reducing condition in the ER [22]. However, despite the obvious influence of the redox environment in protein folding, whether disruption of redox homeostasis promotes protein aggregation is unknown. Here, we addressed this question by exploring the impact of a compromised glutathione redox environment in $C$. elegans models of aggregation-prone proteins. We found that impairment of glutathione homeostasis causes a robust enhancement of phenotypes associated with protein aggregation as a consequence of autophagy disruption and we show this effect is evolutionary conserved from yeast to humans.

\section{Results}

\section{gsr-1 deficiency exacerbates the phenotypes induced by polyQ expression in C. elegans muscle cells}

Although proteotoxic stress may trigger loss of redox homeostasis [22], how impaired redox homeostasis impacts on protein aggregation is unknown. To address this question, we focused on the role of the C. elegans gsr-1 gene, encoding glutathione reductase, for two reasons: first, the glutathione redox system is essential in worms [10, 20], whereas the thioredoxin system is dispensable [14-17]. Second, maternal $g s r-1$ mRNA and/or protein contribution from heterozygous progenitors (balanced with the $\mathrm{qC} 1$ balancer [23]) allows to work with genetically homozygous $g s r-1$ animals, designated as $g s r-1(m+, z-)$, because these animals reach adulthood with no discernible phenotype under normal growth conditions [20], in contrast with gss-1 and $g c s-1$ mutants that are unable to synthesize glutathione and in which maternal contribution only allows growth of the homozygous progeny till L1-L2 larval stages [10]. 
On this premise, we first downregulated $g s r-1$ expression by RNA interference (RNAi) feeding in worms that constitutively express in muscle cells heterologous aggregationprone proteins associated with human neurodegenerative diseases such as A $\beta$ peptide (Alzheimer) [24], $\alpha$-synuclein:: YFP (Parkinson) [25], and Q40::YFP (polyglutamine diseases like Huntington) [26]. Despite to the fact that $A \beta$, $\alpha$-synuclein, and polyQ stretches lack cysteine residues in their sequence, gsr-1 downregulation enhanced the paralysis of $\mathrm{A} \beta$ worms (a readout of $\mathrm{A} \beta$ aggregation) and increased the number of $\alpha$-synuclein::YFP and Q40::YFP aggregates (Supplementary Figure 1a-c). This suggests that the phenotypes caused by $g s r-1$ downregulation are likely not mediated by direct disulfide bond formation on these proteins. Unexpectedly, worms expressing Q40::YFP in muscle cells (from the integrated transgene rmIs133 [Punc$54:: Q 40: \because y f p]$ ) displayed an increasingly penetrant larval arrest phenotype when feeding on subsequent generations of $g s r-1$ RNAi bacteria, an effect that did not occur in transgenic animals expressing the Q35::YFP fusion protein (from the integrated transgene rmIs132 [Punc-54::Q35:: $y f p]$ ) that served as non-aggregating control [26] or in worms expressing the $A \beta$ and $\alpha$-synuclein::YFP proteins (Supplementary Figure 1d-f). Consistent with the RNAi data, $g s r-1\left(m+, z^{-}\right)$animals had a significantly increased number of muscle Q40::YFP aggregates at the L4 (day 3) and young adulthood (day 4) stages (Fig. 1a), which inversely correlated with the muscular performance measured by thrashing activity in liquid medium (Supplementary Figure 2a). No effect was found on the aggregation or thrashing phenotypes of rmIs132 [Punc-54::Q35::yfp]; $g s r-1\left(m+, z^{-}\right)$control worms (Fig. 1a and Supplementary Figure 2b). Transgenic restoration of GSR-1 expression in rmIsl33 [Punc-54::Q40::yfp]; $g s r-1(m+, z-)$ worms decreased the number of Q40::YFP aggregates (Supplementary Figure 2c), demonstrating that the increased aggregation phenotype is specific to gsr-1 deficiency and not to a closely linked unknown mutation (generated by the mutagenesis protocol used to isolate the $g s r-1($ tm3574) deletion and not segregated out by backcrossing with wildtype strain). Furthermore, pharmacological inhibition of GSH synthesis (by buthionine sulfoximine, BSO), GSSG reduction (by 1,3-bis(2-chloroethyl)-1-nitrosourea, BCNU), and GSH depletion (by diethyl maleate, DEM) phenocopied the developmental delay phenotype caused by $g s r-1$ downregulation in worms expressing Q40::YFP fusion protein (Supplementary Figure 2d,e and Fig. 1b). Remarkably, DEM had a much more profound effect than BSO or BCNU as rmIs 133 [Punc-54::Q40::yfp] hermaphrodites exposed to $2.5 \mathrm{mM}$ DEM rapidly became pale and paralyzed and laid very few progeny, which invariably arrested and died at L1-L2 larval stage (Fig. 1b). Together, these data demonstrate that reduced GSR-1 and GSH levels compromise muscle function and survival of worms expressing Q40::YFP in muscle cells.

\section{gsr-1( $m-, z-)$ embryos expressing Q40::YFP in muscle cells undergo dramatic cell blebbing and exploding phenotypes}

We previously reported that $g s r-1(m-, z-)$ embryos, lacking maternal contribution, arrest at the pregastrula/gastrula stage with the embryonic cells superficially normal and no apparent signs of necrosis or apoptosis (Fig. 1c and Movie 1) [20]. In turn, rmIs 133 [Punc-54::Q40::yfp] embryos develop normally until hatching, similar to wildtype embryos (Fig. 1d and Movie 2) [26]. Surprisingly we identified a robust synthetic phenotype in rmIs 133 [Punc-54::Q40::yfp]; gsr-1(m-,z-) embryos as most of these embryos undergo dramatic cell blebbing and/or sudden catastrophic embryo explosion phenotypes when maintained in water at $25^{\circ} \mathrm{C}$ (Figs. 1e, f, Movies 3-4 and Supplementary Table 3$)$. These phenotypes also happened in $g s r-1\left(m-, z^{-}\right)$embryos expressing a Q35::YFP fusion protein from the rmIs132 [Punc-54::Q35::yfp] transgene, although less frequently (Supplementary Table 3). Importantly, when these embryos are maintained in an isotonic buffer, they do not explode but maintain the blebbing phenotype, suggesting the eggshell integrity, which preserves embryonic osmotic homeostasis, is compromised in $g s r-1(m-, z-)$ embryos expressing Q35::YFP or Q40::YFP proteins (Supplementary Table 3).

Under normal conditions, the plasma membrane of eukaryotic cells is tightly bound to an underlying actomyosin cortex that contributes to cell shape maintenance [27]. However, when the actomyosin cortex integrity is disrupted, actin-devoid evaginations (blebs) are generated by internal hydrostatic pressure [28]. To test whether impairment of the actomyosin cortex function might underlay the blebbing phenotype of the rmIs133 [Punc-54::Q40::yfp]; gsr-1(m-,z-) embryos, we examined the dynamics of actin fluorescence markers. The distribution of actin filaments in $r m I s 133$; gsr-1 $(m-, z-)$ embryos did not markedly differ from that of rmIs 133 ; $g s r-1(m+, z+)$ controls and actin was also detected at the sites of blebs (Fig. 1g and Movie 5). As microtubule network destabilization can also result in cell blebbing [29, 30], we next asked whether tubulin dynamics was altered in $r m I s 133$; $g s r-1(m-, z-)$ embryos. Using tubulin fluorescence markers, we identified an aberrant distribution of the tubulin filaments in the periphery of the $r m I s 133 ; g s r-1\left(m-z^{-}\right)$embryonic cells that is not observed in control embryos (Fig. 1h and Movie 6). Similar results were obtained with tubulin immunostaining (Supplementary Figure 3). These data suggest that, in a GSH compromised environment, polyQ proteins modify the dynamics of the microtubule network, impinging on plasma membrane integrity, and function. 

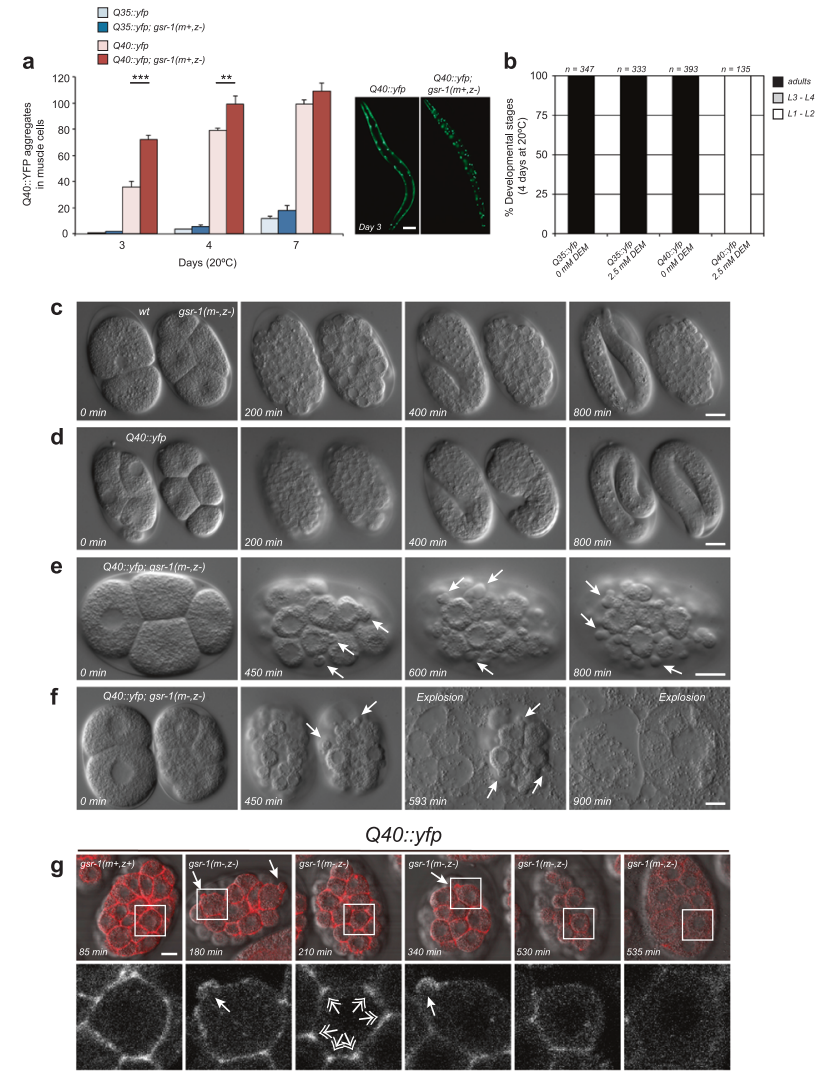
Q40::yfp

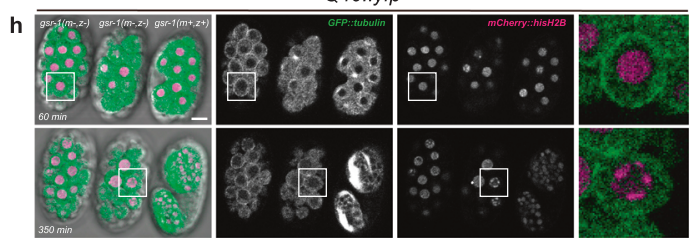

\section{gsr-1 mutants show enhanced polyQ proteins aggregation in neurons and intestinal cells}

To determine whether the protective effect of GSR-1 in polyQ worms may be restricted to muscle cells or extended to other tissues, we analyzed $g s r-1\left(m+, z^{-}\right)$animals expressing polyQ proteins in neuronal and intestinal cells. In addition, to pharmacologically validate the data obtained with $\operatorname{gsr}-1\left(m+, z^{-}\right)$mutants in these models, we opted for depleting the pool of reduced glutathione by DEM treatment as it produced the more penetrant phenotype on rmIs133 [Punc-54::Q40::yfp] worms (Fig. 1b). First, we used a $C$. elegans model of Machado-Joseph disease pathogenesis in which mutant human Ataxin-3 (ATXN3) fused to YFP is expressed in a pan-neuronal fashion [31]. Similar to muscular polyQ models, we found that the $g s r-1$ $\left(m+, z^{-}\right)$mutation caused a robust increase of ventral nerve cord fluorescent foci (the location where these foci are more discretely distributed and, therefore, more easily quantified) in worms expressing mutant ATXN3::Q130::YFP but not in
Fig. 1 Phenotypes of the $g s r-1$ mutation and diethyl maleate (DEM) treatment on $C$. elegans expressing Q40::YFP protein in muscle cells. a The $g s r-1\left(m+, z^{-}\right)$mutation increases the number of Q40::YFP, but no Q35::YFP, aggregates in worm muscle cells. Data are the mean $\pm \mathrm{S}$. E.M. of three independent experiments $(n=10$ animals per strain and assay). ${ }^{*} p<0.01 ; * * * p<0.001$ by unpaired, two-tailed Student's $t$ test. Images show one representative example of muscle Q40::YFP aggregates for each genotype. Scale bar $100 \mu \mathrm{m}$. b $2.5 \mathrm{mM}$ DEM treatment causes a fully penetrant L1-L2 larval arrest on worms expressing a Q40::YFP fusion protein in muscle cells while no effect is found in $Q 35:: y f p$ animals. Data are from three independent experiments ( $n=$ total number of animals assayed). c-f Differential interference contrast still images of developing wild-type and $g s r-1\left(m-, z^{-}\right)$ embryos (c), Q40::yfp embryos (d) and $Q 40:: y f p ; \quad g s r-1(m-, z-)$ embryos (e, f). Arrows indicate transient blebs. Embryo explosion in (f) is denoted by the sudden disappearance of embryonic cells. The still images in c-f are taken from Movies 1 to 4, respectively. g Control $g s r$ $1(m+, z+)$ and $Q 40: \because y f p ; g s r-1\left(m-, z^{-}\right)$embryos expressing the actin marker LifeAct::mCherry were mounted and recorded together. Bottom row shows higher magnification images of LifeAct::mCherry only. Closed arrows point to examples of membrane blebs whereas double-headed arrows indicate F-actin accumulation at cell vertices. Last frame corresponds to the moment when the eggshell suddenly fills completely and the embryo explodes. The still images are taken from Movie 5. h Control $Q 40:: y f p ; g s r-1(m+, z+)$ and $Q 40:: y f p ; g s r-1(m-, z-)$ embryos expressing the microtubule marker GFP::tubulin and the chromatin marker mCherry::hisH2B (green and magenta in merge, respectively) were mounted and recorded together. Right column shows higher magnification images of boxed area. Strong accumulation of microtubules at the plasma membrane is observed at $60 \mathrm{~min}$ (top row). Condensation of chromatin at the nuclear periphery occurs at $350 \mathrm{~min}$. Additional examples are provided in Movie 6. All movie recordings were carried out at $25^{\circ} \mathrm{C}$ in $\mathrm{H}_{2} \mathrm{O}$ except in (h) that was performed in $\mathrm{M} 9$ buffer at $25^{\circ} \mathrm{C}$. Time is indicated from start of recording. Scale bar $10 \mu \mathrm{m}$

ATXN3::Q75::YFP worms that served as non-aggregating controls (Fig. 2a). However, upon exposure of animals to DEM, we observed an inverse correlation with toxicity as the ATXN3::Q75::YFP control worms suffered a significant developmental delay while, in contrast, ATXN3::Q130:: YFP worms developed at the same rate compared with untreated controls (Fig. 2b). These results suggest that a protective mechanism might operate in neurons when a certain aggregation threshold is surpassed.

We further confirmed the deleterious effect of the $g_{s r-1}$ mutation on neuronal polyQ toxicity using worms that express in the six touch receptor neurons the first 57 amino acids of the human Huntingtin protein followed by normal (Q19) or expanded (Q128) polyglutamines and that is fused to Cyan fluorescent protein (CFP) [32]. Although the gsr-1 mutation did not modify light touch response of worms expressing the non-aggregating HTT57::Q19::CFP fusion protein control, we observed a significant decrease of the touch response in HTT57::Q128::CFP nematodes (Fig. 2c). In contrast to the ataxin model, we did not identify any differential growth response of HTT57::Q19::CFP and HTT57:: Q28::CFP worms to DEM treatment (Fig. 2d), most likely due to the dispensability of touch neurons for development. 


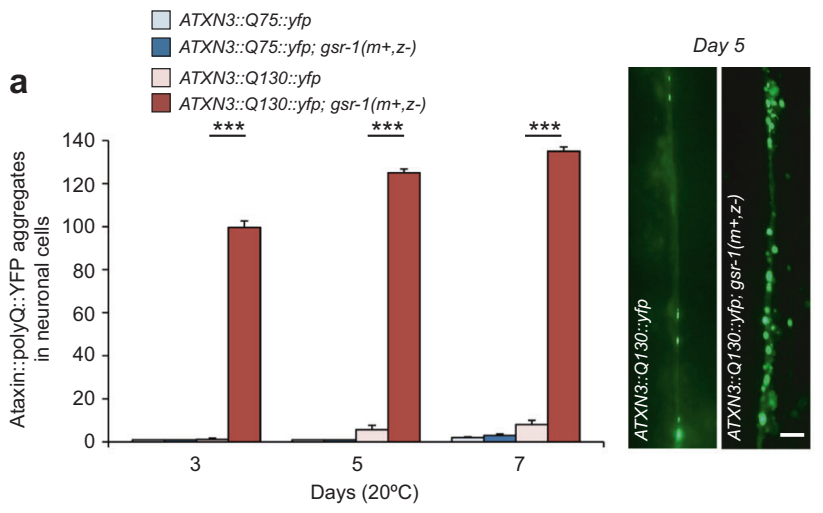

b

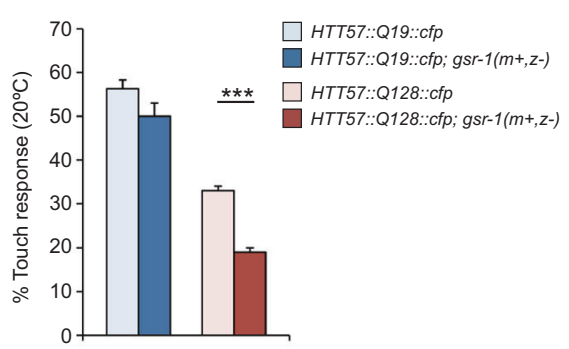

d

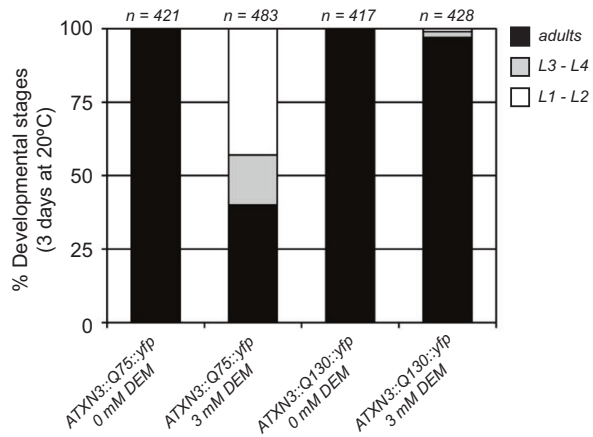

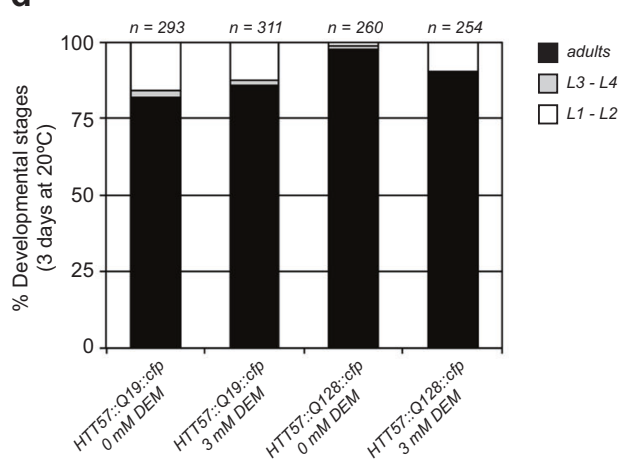

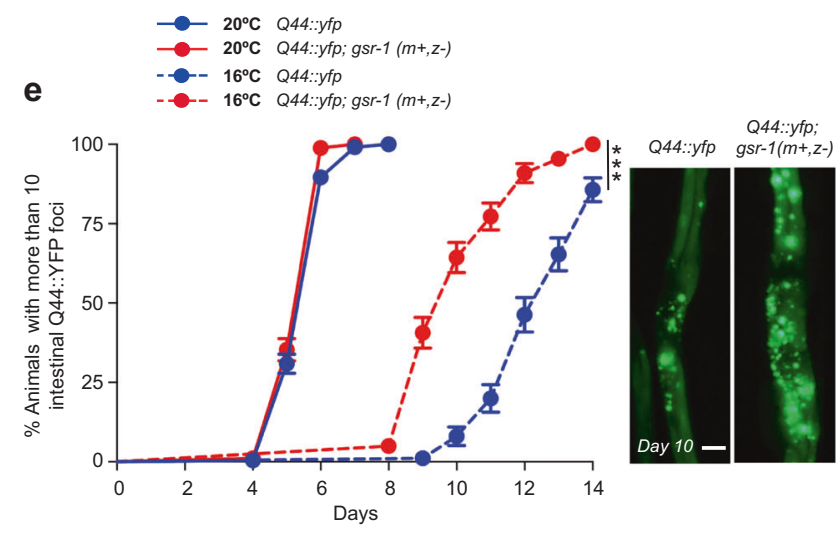

f

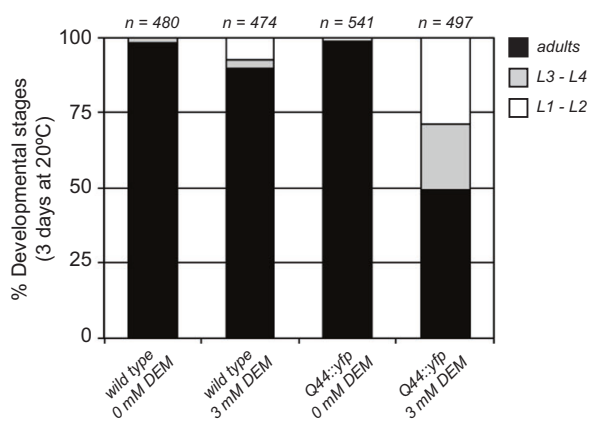

Fig. 2 Phenotypes of the gsr-1 mutation and diethyl maleate (DEM) treatment on $C$. elegans expressing polyQ proteins in neurons and intestinal cells. a The $g s r-1\left(m+, z^{-}\right)$mutation increases the number of ATXN3::Q130::YFP aggregates in the worm ventral nerve cord while no effect is found in control worms expressing ATXN3::Q75:: YFP fusion protein. Data are the mean \pm S.E.M. of three independent experiments $(n=12$ animals per strain and assay). $* * * p<0.001$ by unpaired, two-tailed Student's $t$-test. Images show one representative example of ventral nerve cord ATXN3::Q130::YFP aggregates for each genotype. Scale bar $20 \mu \mathrm{m}$. b $3 \mathrm{mM}$ DEM treatment delays the larval development of worms expressing pan-neuronal ATXN3:: Q75::YFP but not ATXN3::Q130::YFP fusion proteins. Data are from three independent experiments $(n=$ total number of animals assayed). c Percentage of touch-responsiveness of $w t$ versus $g s r-1(\mathrm{~m}$ ,$\left.+ z^{-}\right)$worms expressing HTT57::Q19::CFP and HTT57::Q128::CFP fusion proteins in mechanosensory neurons. Data are the mean $\pm \mathrm{S}$.

D. of two independent experiments $(n \geq 100$ animals per strain and assay). $* * * p<0.001$ by one-way analysis of variance (ANOVA) with Tukey's multiple comparison test. d 3 mM DEM treatment does not affect the larval development of worms expressing HTT57:: Q19::CFP and HTT57::Q128::CFP fusion proteins in mechanosensory neurons. Data are from three independent experiments ( $n=$ total number of animals assayed). e Onset of appearance of YFP fluorescent aggregates in $w t$ versus $g s r-1(m+, z-)$ worms expressing Q44::YFP fusion protein as function of time. Data are the mean \pm S.E.M of three independent experiments $(n=50$ animals per strain and assay). ${ }^{* *} p<0.001$ by log-rank (Mantel-Cox) test. Images show one representative example of intestinal Q44::YFP aggregates for each genotype. Scale bar $50 \mu \mathrm{m}$. f $3 \mathrm{mM}$ DEM treatment delays the larval development of worms expressing a Q44::YFP fusion protein in intestinal cells. Data are from three independent experiments ( $n=$ total number of animals assayed) 
Finally, we also assayed the impact of the $g s r-1$ mutation in worms expressing a Q44::YFP fusion protein in intestinal cells [33]. At $20^{\circ} \mathrm{C}$, the appearance onset of intestinal fluorescent aggregates was similar in both wild-type and gsr- $1\left(m+, z^{-}\right)$backgrounds (Fig. $\left.2 \mathrm{e}\right)$. However, at $16^{\circ} \mathrm{C}$, the formation of intestinal Q44::YFP aggregates occurred earlier and these aggregates were bigger and more abundant in gsr-1 $\left(m+, z^{-}\right)$worms compared with wild-type controls (Fig. 2e). In consonance, animals expressing Q44::YFP proteins were highly sensitive to DEM (Fig. 2f). Collectively, these data indicate that $g s r-1$ and GSH deficiency enhance polyQ proteins aggregation regardless of the cell type or tissue of expression.

\section{gsr-1 deficiency is deleterious in C. elegans expressing human $A \beta$ and $\alpha$-synuclein proteins}

In addition to the effect on polyQ proteins aggregation, the previously mentioned enhanced paralysis of worms expressing human $\mathrm{A} \beta$ or increased $\alpha$-synuclein::YFP aggregation in animals fed with gsr-1 RNAi (Supplementary Figure 1a,b) pointed to a general effect of $g s r-1$ in proteostasis. We then characterized the effect of the gsr-1 mutation in these two additional models of protein aggregation. Worms expressing the $d v I s 2$ [Punc-54::A $\beta$; rol-6 (su1006)] transgene produce human $\mathrm{A} \beta$ protein in muscle cells where it aggregates and causes age-dependent progressive paralysis at $20^{\circ} \mathrm{C}$ but not (or very mildly) at the $16{ }^{\circ} \mathrm{C}$ permissive temperature [24]. As shown in Fig. 3a, the $g s r-1\left(m+, z^{-}\right)$mutation induced robust paralysis in $d v I s 2$ $[$ Punc-54::A $\beta]$ worms at the permissive temperature. This synthetic interaction was phenocopied pharmacologically with DEM, causing a complete L1-L2 larval arrest phenotype of $d v I s 2$ worms (Fig. 3b). Moreover, $d v I s 2$ [Punc-54:: $A \beta]$; $g s r-1\left(m-, z^{-}\right)$embryos also showed blebbing and explosion phenotypes (Supplementary Table 3), similar to gsr-1(m-,z-) embryos expressing Q40::YFP under the control of the same muscle specific unc-54 promoter (Figs. 1e, f). Interestingly, an even more striking phenotype was found when combining the $g s r-1\left(m+, z^{-}\right)$mutation with a different A $\beta$ transgene, $d v I s 14$ [Punc-54::A $;$; Pmtl-2::gfp] [34]. In this case, $d v I s 14 ; g s r-1\left(m+, z^{-}\right)$worms raised at the permissive temperature displayed a much faster paralysis (50\% paralyzed animals at day 8.5) as compared with that of $d v I s 2 ; \operatorname{gsr}-1\left(m+, z^{-}\right)$counterparts $(50 \%$ paralyzed animals at day 11.5) (Fig. 3c versus Fig. 3a). In addition, dvIs14; $g s r-1\left(m+, z^{-}\right)$animals showed a fully penetrant egg-laying phenotype, most likely reflecting the lack of contractility of the uterine and vulva muscles, which are responsible for egg extrusion (Fig. 3d). The genetic interaction between $\mathrm{A} \beta$ protein and the $g s r-1$ mutation was also found in worms expressing human $\mathrm{A} \beta$ in the nervous system from the gnaIs 2 [Punc-119::A $\beta$ ] transgene [35]. Thus, the $g s r-1\left(m+, z^{-}\right)$ mutation causes a strong developmental delay in a gnaIs 2 genetic background (Fig. 3e), which correlated with a higher sensitivity to DEM of gnals2 nematodes (Fig. 3f).

Similarlly, the aggregation in muscle cells of the $\alpha$ synuclein::YFP fusion protein expressed from the $p k I s 2386$

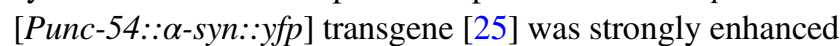
in $g s r-1\left(m+, z^{-}\right)$mutants (Fig. 4a) although, in contrast to animals expressing Q40::YFP or A $\beta$ in muscle cells, DEM treatment did not cause any developmental phenotype in muscle $\alpha$-synuclein::YFP animals (Fig. 4b). Similarly, in

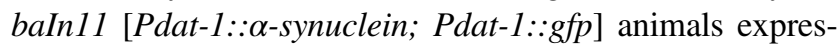
sing $\alpha$-synuclein in the six worm head dopaminergic neurons [36], the $g s r-1\left(m+, z^{-}\right)$mutation significantly increased neurodegeneration in an age-dependent manner (Fig. 4c), whereas no phenotype was observed when these animals are treated with DEM (Fig. 4d). The lack of any deleterious effect of DEM treatment in worms expressing $\alpha$-synuclein:: YFP in muscle cells may be due to the very mild effect on motility, which has been reported for these worms [25] in comparison with human A $\beta$ or Q40::YFP expressing animals [24, 26]. Similarly, the dispensability of dopaminergic signaling for worm survival [37] may explain the absence of deleterious phenotypes of worms expressing $\alpha$-synuclein in dopaminergic neurons when exposed to DEM.

\section{gsr-1 deficiency worsens the phenotypes of $C$. elegans expressing endogenous metastable proteins}

All models so far described in this work express heterologous aggregation-prone proteins (A $\beta$, a-synuclein::GFP or polyQ::GFP) not naturally found in $C$. elegans. To rule out that the effect of the $g s r-1(m+, z-)$ mutation in these models might be a consequence of expressing foreign proteins in worm cells, we monitored mutant phenotypes in animals bearing thermosensitive alleles of endogenous genes, which encode metastable proteins that are highly sensitive to changes in the protein folding environment. To this end, we used unc-52(e669su250) and let-60(ga89) mutants that display a highly penetrant paralysis or embryonic arrest phenotype, respectively, at the non-permissive temperature of $25^{\circ} \mathrm{C}$ but are roughly wild-type at the permissive temperature of $16^{\circ} \mathrm{C}$ [38]. Consistent with the results above, the double mutant unc-52(e669su250); $g s r-1(m+, z-)$ displayed a robust paralysis phenotype at $16^{\circ} \mathrm{C}$ (Fig. 4e). This synthetic interaction was phenocopied by treatment of unc52(e669su250) mutants with $2 \mathrm{mM}$ DEM, causing a fully penetrant early larval arrest (Fig. 4f). Similarly, a strong synthetic interaction was found between let-60(ga89) and gsr-1 mutants as shown by the impossibility to generate viable progeny from let-60(ga89); gsr-1/qCl::rfp balanced animals at permissive temperature (Fig. $4 \mathrm{~g}$ ). To rule out a possible deleterious effect of the $q C 1:: r f p$ balancer, we stabilized the $g s r$-1(tm3574) mutation with a different 

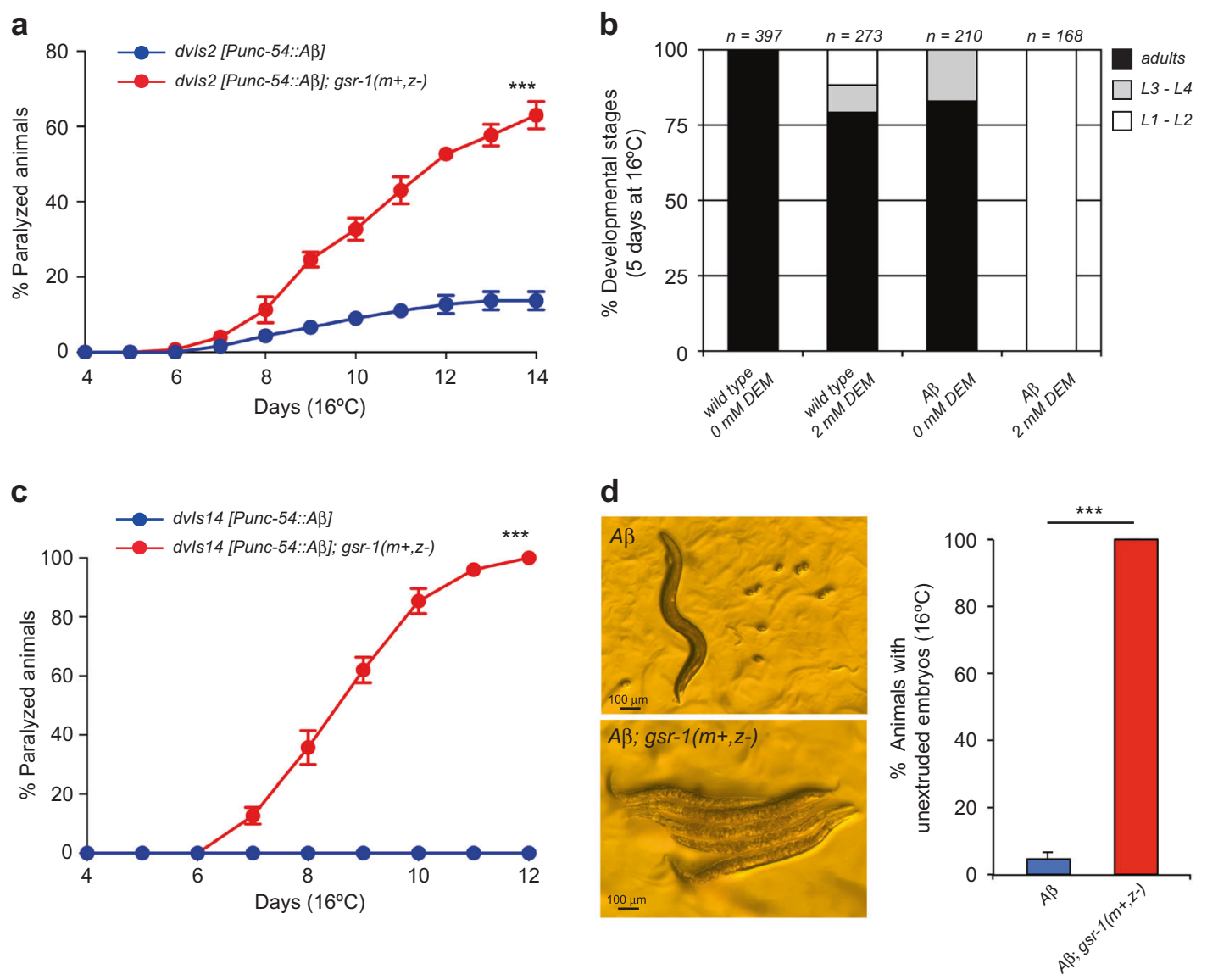
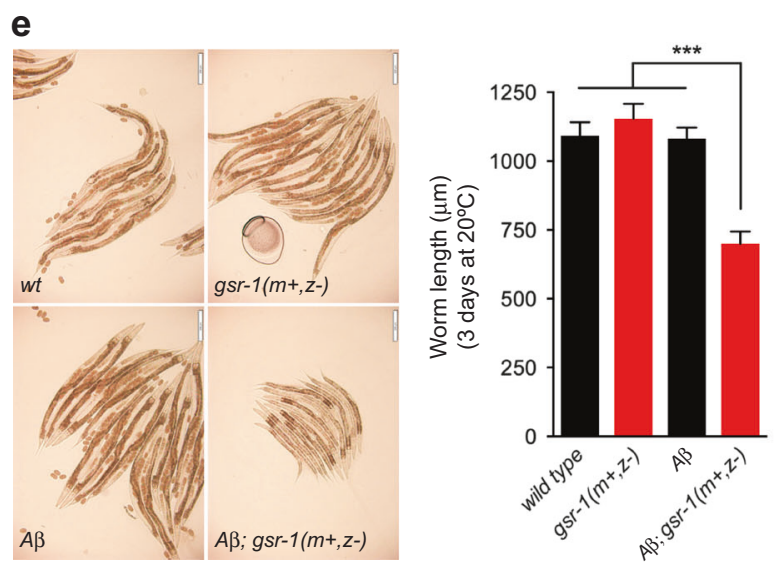

Fig. 3 Phenotypes of the gsr-1 mutation and diethyl maleate (DEM) treatment on $C$. elegans expressing the human $\beta$-amyloid peptide. a The $g s r-1(m+, z-)$ mutation increases the paralysis of worms expressing the human $\beta$-amyloid peptide in muscle cells from the $d v I s 2$ integrated array. Data are the mean \pm S.E.M. of three independent experiments $(n=25$ animals per strain and assay). $* * * p<0.001$ by log-rank (Mantel-Cox) test. b $2 \mathrm{mM}$ DEM treatment causes a fully penetrant L1-L2 larval arrest on worms expressing the human $\beta$-amyloid peptide in muscle cells. Data are from three independent experiments ( $n=$ total number of animals assayed). $\mathbf{c}$ When expressing the human $\beta$-amyloid peptide in muscle cells from the $d v I s 14$ integrated array, the $g s r-1\left(m+, z^{-}\right)$mutation increases the paralysis of worms much faster than that observed in $d v I s 2$ animals. Data are the mean \pm S.E.M. of three independent experiments $(n=25$ animals per strain and assay). ${ }^{* * *} p<0.001$ by log-rank (Mantel-Cox) test. $\mathbf{d}$ The f

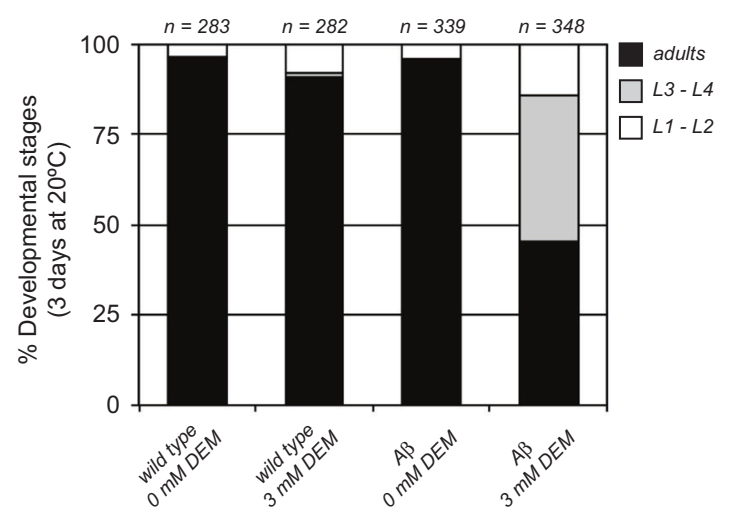

$g s r-1(m+, z-)$ mutation provokes a fully penetrant unextruded embryo phenotype in worms expressing the human $\beta$-amyloid peptide in muscle cells from the $d v I s 14$ transgene. Data are the mean \pm S.E.M. of three independent experiments ( $n=50$ animals per strain and assay). $* * * p<0.001$ by unpaired, two-tailed Student's $t$-test. Images show one representative example of $d v I s 14$ and $d v I s 14 ; g s r-1(m+, z-)$ worms. Scale bar $100 \mu \mathrm{m}$. e The $g s r-1\left(m+, z_{-}\right)$mutation delays the development of worms expressing the human $\beta$-amyloid peptide in the nervous system. Data are the mean \pm S.E.M. of three independent experiments ( $n=40$ worms per strain and assay). $* * * p<0.001$ by unpaired, twotailed Student's $t$-test. Images show one representative example for each genotype. Scale bar $200 \mu \mathrm{m}$. f $3 \mathrm{mM}$ DEM treatment delays the larval development of worms expressing the human $\beta$-amyloid peptide in neurons. Data are from three independent experiments $(n=$ total number of animals assayed) 
a

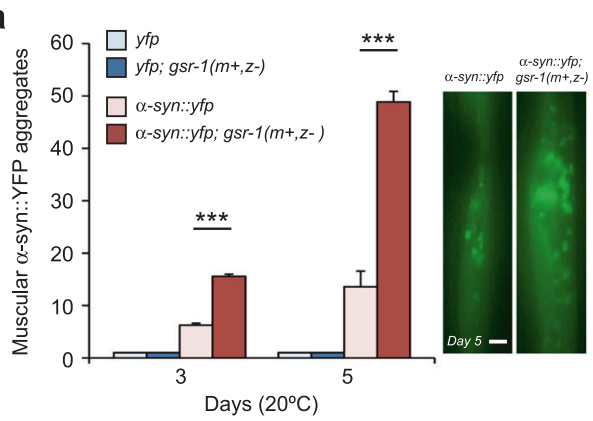

C

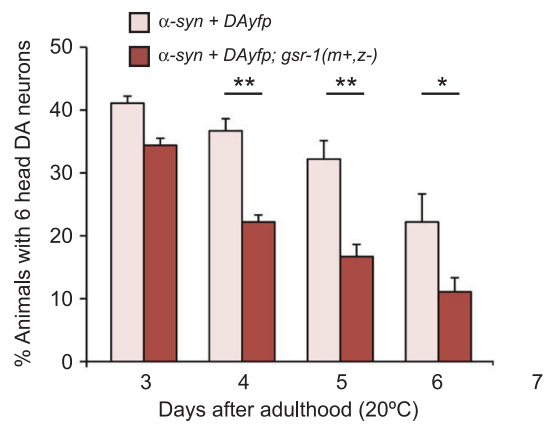

b

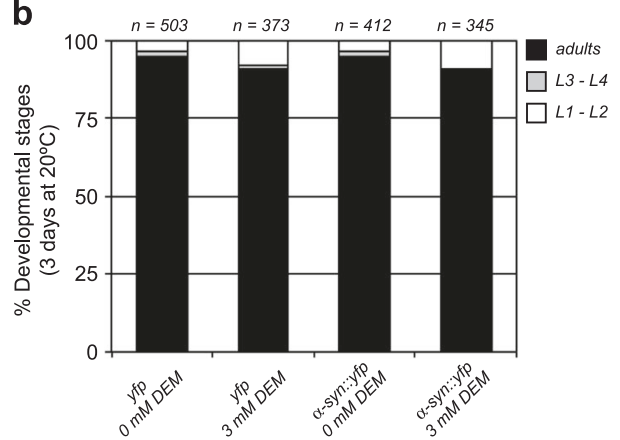

d

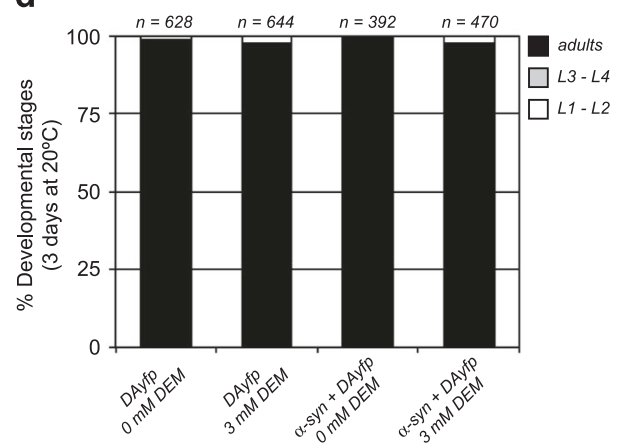

e

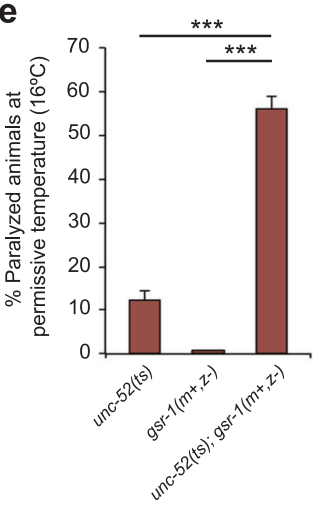

g

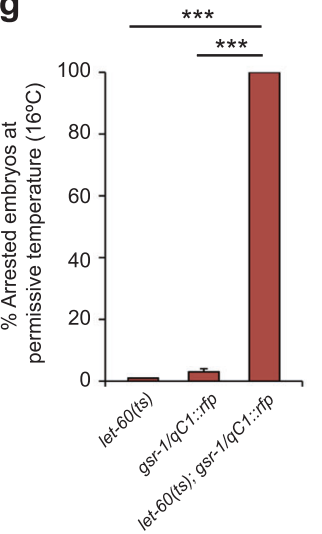

f

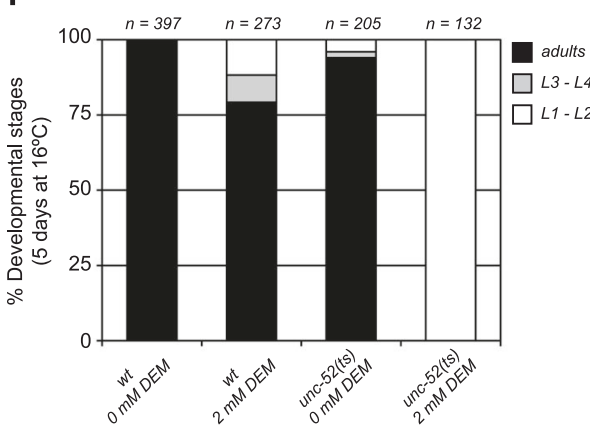

h

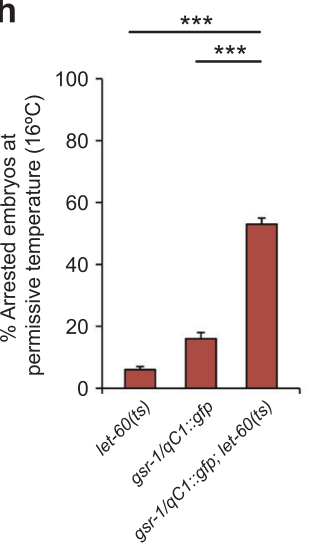

i

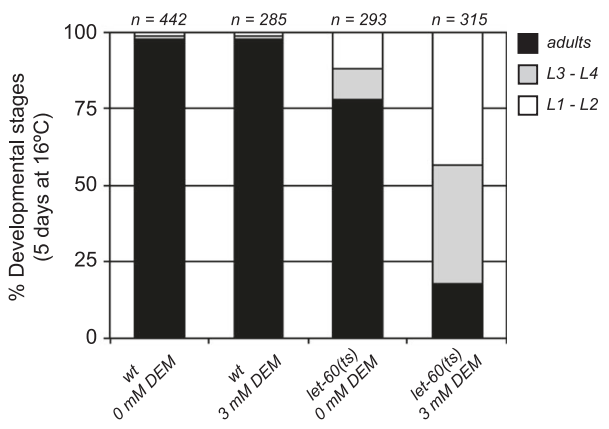

version of the $q C l$ balancer tagged with GFP (see Supplementary Table 1 for full genomic information of both balancers) that resulted in $50 \%$ viable progeny in a let-60 (ga89) background (Fig. 4h). Remarkably, none of this viable progeny was $g s r-1\left(m+, z^{-}\right)$, but all $g s r-1(t m 3574)$ / $q C 1:: g f p$, thus confirming the synthetic lethal phenotype of 
Fig. 4 Phenotypes of the gsr-1 mutation and diethyl maleate (DEM) treatment on $C$. elegans expressing human $\alpha$-synuclein and endogenous metastable proteins. a The $g s r-1\left(m+, z^{-}\right)$mutation increases the number of $\alpha-\mathrm{SYN}::$ YFP aggregates in the muscle cells. Aggregate quantification was restricted to aggregates located between the two pharyngeal bulbs. Data are the mean \pm S.E.M. of three independent experiments $\left(n=12\right.$ animals per strain and assay). ${ }^{* * *} p<0.001$ by unpaired, two-tailed Student's $t$-test. Images show one representative example of muscle $\alpha-S Y N:$ YFP aggregates for each genotype. Scale bar $10 \mu \mathrm{m}$. b $3 \mathrm{mM}$ DEM treatment does not affect the development of worms expressing the $\alpha$-SYN::YFP fusion protein in muscle cells. Data are from three independent experiments $(n=$ total number of animals assayed). c The $g s r-1\left(m+, z^{-}\right)$mutation accelerates the agedependent neurodegeneration of dopaminergic neurons expressing human $\alpha$-synuclein. Data are the mean \pm S.E.M. of three independent experiments $(n=30$ animals per strain and assay). $* p<0.05$, $* * p<$ 0.01 by unpaired, two-tailed Student's $t$-test. d $3 \mathrm{mM}$ DEM treatment does not affect the development of worms expressing human $\alpha$-synuclein in dopaminergic neurons. Data are from three independent experiments ( $n=$ total number of animals assayed). e The $g s r-1\left(m+, z_{-}\right)$ mutation increases the paralysis phenotype of unc-52(e669su250) worms at permissive temperature. Data are the mean \pm S.E.M. of three independent experiments $(\mathrm{n} \geq 70$ animals per strain and assay, scored at day 7 after egg-lay). ***p $<0.001$ by unpaired, two-tailed Student's $t$ test. f $2 \mathrm{mM}$ DEM treatment causes a fully penetrant L1-L2 larval arrest on unc-52(e669su250) worms at permissive temperature. Data are from three independent experiments $(n=$ total number of animals assayed). $\mathbf{g}, \mathbf{h}$ The $g s r-1\left(m+, z^{-}\right)$mutation increases the embryonic arrest phenotype of let-60(ga89) worms at permissive temperature. g let-60(ga89); gsr-1/qC1::rfp worms produced only arrested embryos while (h) let-60(ga89); $g s r-1 / q C 1:: g f p$ worms only produced both balanced viable progeny and arrested embryos but not unbalanced $g s r$ $1\left(m+, z^{-}\right)$progeny. Data are the mean \pm S.E.M. of three independent experiments $(n \geq 100$ embryos/animals per strain and assay). $* * * p<$ 0.001 by unpaired, two-tailed Student's $t$-test. i $3 \mathrm{mM}$ DEM treatment delays the larval development of let-60(ga89) worms at permissive temperature. Data are from three independent experiments $(n=$ total number of animals assayed)

$g s r-1\left(m+, z^{-}\right)$and let-60(ga89) mutants, which was further supported by the larval arrest phenotype of let-60(ga89) animals in the presence of $3 \mathrm{mM}$ DEM (Fig. 4i). Together, these data suggest that glutathione reductase and the maintenance of a proper GSH redox homeostasis may protect against protein aggregation.

\section{Autophagy function is impaired in gsr-1 mutants}

Proteostasis is maintained by a delicate equilibrium of different molecular pathways. Some of these pathways are at the core of the proteome maintenance such as protein synthesis, folding, trafficking, or degradation, while others act as modifiers of proteostasis such as UPR, nutritional and metabolic status, stress situations, genetic and epigenetic susceptibility, or physiological processes like aging [2]. To ascertain which of these pathways might underlay the protective effect of GSR-1 and GSH in protein aggregation, we first tested the effect of $g s r-1$ deficiency on different reporters of proteostasis regulators including insulin, oxidative stress, UPR-ER, heat-shock, proteasome, mitophagy, or autophagy pathways, under basal and stress conditions (Supplementary Figure 4 and Fig. 5a, b). We found that the $g s r-1\left(m+, z^{-}\right)$mutation did not interfere with the insulin, heat-shock, proteasome, and mitophagy pathways (Supplementary Figure 4a, d-i). However, we observed an induction of the oxidative stress reporter SOD-3::GFP under basal, but not stressed conditions (Supplementary Figure $4 \mathrm{~b}$ ). As SOD-3::GFP induction was not accompanied by an increase in DAF-16::GFP nuclear translocation (Supplementary Figure 4a), the effect of the $g s r-1$ mutation on sod-3 transcription is more likely due to a DAF16-independent response to oxidative stress rather than a role in proteostasis. In addition, the $g s r-1\left(m+, z^{-}\right)$mutation caused a mild downregulation of the UPR-ER reporter HSP-4::GFP under both conditions (Supplementary Figure 4c).

In contrast to all reporters described above, we identified a clear effect of the $g s r-1$ mutation in worms carrying the sqIs 17 transgene, which expresses the HLH-30::GFP autophagy marker [39]. HLH-30 is the worm orthologue of the mammalian TFEB transcription factor and, under normal growth conditions, these transcription factors are inactive and diffusely distributed in the cytoplasm. However, upon stress conditions such as starvation and heatshock, HLH-30/TFEB translocate into the nucleus, triggering the transcription of genes required for autophagy induction and lysosomal biogenesis [39-42]. We found that the cytosolic subcellular localization of HLH-30::GFP was not modified in a $g s r-1\left(m+, z^{-}\right)$mutant background in wellfed animals (Fig. 5a). In turn, 1-h starvation or $30^{\circ} \mathrm{C}$ heatshock was enough to induce a strong nuclear translocation of the HLH-30::GFP reporter, a phenomenon severely hampered in $g s r-1(m+, z-)$ worms subjected to starvation, but not heat-shock (Fig. 5a and Supplementary Figure 5a).

These results suggested that gsr-1 deficiency may negatively impact on proteostasis by impairing autophagydependent protein degradation, at least upon specific stimuli-like starvation. To test for this possibility, we used worms that carry the bpIs151 transgene and express the SQST-1::GFP reporter protein, which is typically degraded by autophagy in non-stressed conditions but accumulates in the form of fluorescent foci, mainly in embryonic and larval intestinal cells, when autophagy is compromised [43]. Consistently, we found no SQST-1::GFP foci in bpIs151; $g s r-1\left(m+, z^{-}\right)$embryos and L1 larvae under normal growth conditions (Fig. 5b and Supplementary Figure 5b). However, when bpIs151; gsr-1 $\left(m+, z^{-}\right)$worms were starved for $24 \mathrm{~h}$, we observed a robust increase in the number of SQST$1::$ GFP foci in $g s r-1\left(m+, z^{-}\right)$embryos (also accompanied by the appearance of large vacuoles in some embryos) (Fig. 5b). Similar results were obtained when bpIs151; gsr$l(m+, z-)$ animals were subjected to a mild impairment of autophagy by RNAi downregulation of lgg-1/LC3 (required for autophagosome (AP) biogenesis), which resulted in a 
a
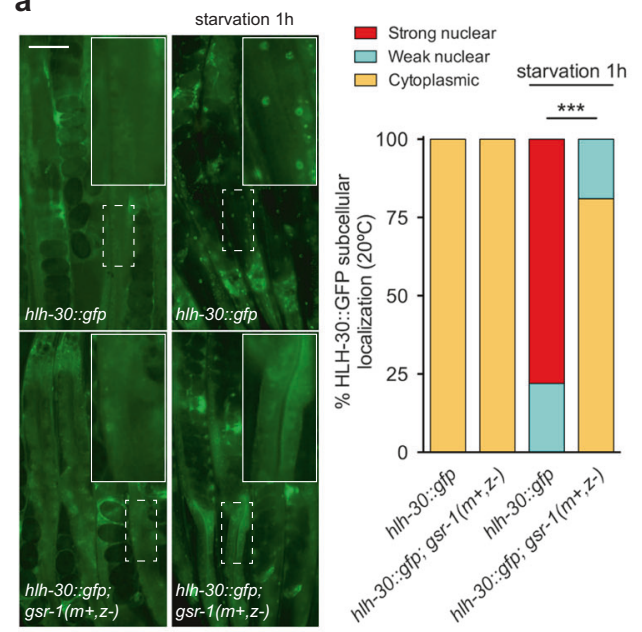

b

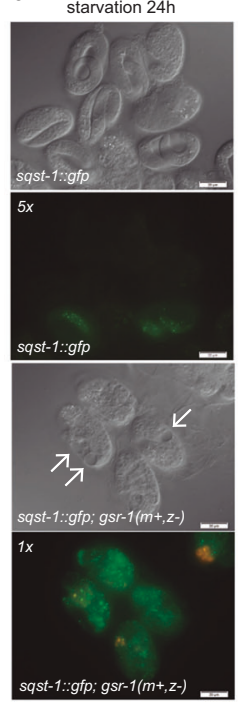

c

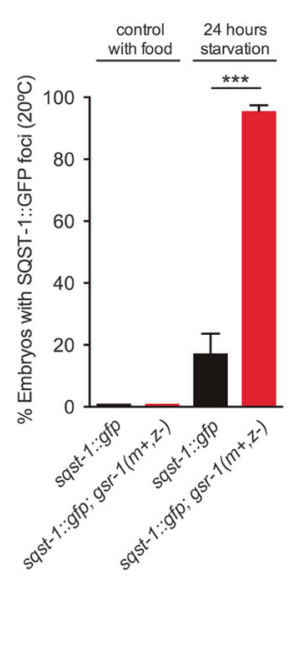

$\rightarrow \operatorname{atg}-3$

$-g s r-1(m+, z-)$

$\rightarrow$ unc-52

- $g S r-1(m+, 7-) \cdot 4 n c-52$

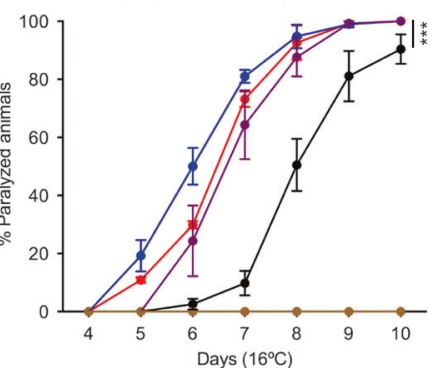

d

e

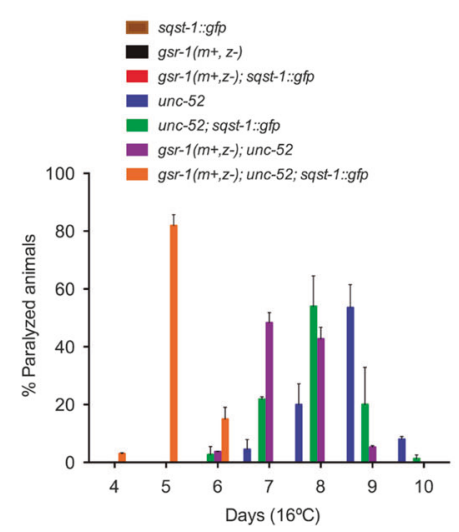

Fig. 5 The gsr-1 mutation enhances the phenotypes of $C$. elegans proteostasis models by preventing autophagy function. a The $g s r-1$ $(m+, z-)$ mutation does not modify the diffuse cytoplasmic localization of the HLH-30::GFP reporter under well-fed conditions but strongly inhibits its nuclear translocation after one hour starvation. Data are the mean of three independent experiments $(n=50$ animals per strain and assay). $* * * p<0.001$ by chi ${ }^{2}$ test. Scale bar $50 \mu \mathrm{m}$. b The $g s r-1(m+, z-)$ mutation increases the appearance of SQST-1::GFP foci in embryos generated by parents that have been starved for $24 \mathrm{~h}$. DIC images are provided for embryo position identification. The exposure time for the fluorescence images of sqst-1::gfp embryos was 5x times of that of sqst-1::gfp; $g s r-1(m+, z-)$ embryos. Arrows indicate vacuoles in the sqst-1::gfp; gsr-1(m+,z-) embryos. Scale bar $20 \mu \mathrm{m}$. Data are the mean \pm S.D. of two independent experiments $(n=50$ embryos per strain and assay). $* * * p<0.001$ by unpaired, two-tailed Student's $t$-test. c The atg-3(bp412) mutation increases the paralysis of unc-52 (e669su250) worms, expressing metastable UNC-52 protein in muscle cells. Data are the mean \pm S.E.M. of three independent experiments
( $n \geq 50$ animals per strain and assay). *** $p<0.001$ by log-rank (Mantel-Cox) test of all strains carrying the unc-52(e669su250) allele versus the unc-52 single mutant control. d Expression of the SQST-1:: GFP autophagy substrate increases the paralysis onset of unc-52 (e669su250) worms and this effect is further enhanced in a $g s r-1(m+, z-)$ background. Data are the mean \pm S.E.M. of three independent experiments ( $n \geq 50$ animals per strain and assay). $p<0.001$ by $\log$ rank (Mantel-Cox) test of all strains carrying the unc-52(e669su250) allele versus the unc-52 single mutant control. e Hypodermal seam cells expressing the mCherry::GFP::LGG-1 tandem reporter, imaged at day 1 of adulthood. Autophagosomes (APs) are labeled in yellow while autolysosomes (AL) are labeled in red. Data are the mean \pm S.E. M. of four independent experiments $(n \geq 12$ animals per strain and assay, with two seam cells quantified for each animal). $* p<0.05$, $* * p<0.01, * * * p<0.001$ by one-way analysis of variance (ANOVA) with Tukey's multiple comparison test, compared with their respective AP or AL controls. Scale bar $10 \mu \mathrm{m}$ significant increase in the number of $g s r-1(m+, z-)$ L1 larvae with SQST-1::GFP foci (Supplementary Figure $5 \mathrm{~b}$ ).

Next, we reasoned that if $g s r-1$ mutants enhance the phenotypes of worm models of proteostasis by impairing autophagy, then genetically blocking autophagy in these proteostasis models would similarly result in an enhancement of their deleterious phenotypes. To our knowledge, no reports have so far addressed the implication of autophagy 
in the phenotypes of unc-52 or let-60 thermosensitive mutants while a role for autophagy on the phenotypes of worms expressing human $\mathrm{A} \beta, \alpha$-synuclein and polyQ proteins has only been studied by RNAi downregulation [42, 44-46]. To provide more compelling evidence of the implication of autophagy in these proteostasis models, we employed atg-3(bp412) mutants, in which autophagy is blocked at the AP formation step [43]. We corroborated the RNAi results in the muscle $A \beta, \alpha$-synuclein, and polyQ worm models and found an enhancement of their respective paralysis or aggregation phenotypes in an atg-3 background (Supplementary Figure 5c-e). Interestingly, we also found a significant enhancement of the paralysis in unc-52; atg-3 double mutants compared with unc-52 controls at permissive temperature (Fig. 5c), a result that was phenocopied using lgg-1 RNAi (Supplementary Figure 5f). We also attempted to evaluate the effect of the atg-3(bp412) mutation in the embryonic arrest phenotype of let-60(ga89) mutants but failed to isolate a double mutant atg-3; let-60, which likely indicates a lethal interaction. In support of this possibility, let-60(ga89) mutants expressing the autophagy substrate SQST-1::GFP display a 10-fold increase in their embryonic arrest phenotype at the permissive temperature (Supplementary Figure 5g). Together, these data reveal that the endogenous, aggregation-prone metastable UNC-52 and LET-60 proteins are likely degraded by autophagy and reinforce the implication of autophagy in dismissing heterologous $\mathrm{A} \beta, \alpha$-synuclein and polyQ proteins in worm models of neurodegenerative diseases.

Glutathione depletion has been shown to increase protein carbonylation and aggregation [47]. We hypothesized that the deleterious effect of the $g s r-1$ mutation on proteostasis could be explained by an increase of the load of aggregated proteins deemed for degradation, ultimately resulting in a collapse of autophagy. To test this hypothesis, we aimed to generate atg-3(bp4l2); $g s r-1\left(m+, z^{-}\right)$double mutants in the different protein aggregation models and found strong synthetic interactions. For instance, we were unable to generate viable atg-3; $g s r-1(m+, z-)$ animals expressing human $\mathrm{A} \beta$ protein in muscle cells (either from $d v I s 2$ or $d v I s 14$ transgenes). In consonance, although viable, atg-3; $g s r-1(m+, z-)$ worms expressing $\alpha$-SYN::GFP in muscle cells were sick and displayed a high percentage of internal hatching, which precluded proper quantification of paralysis. Similarly, atg-3; $g s r-1\left(m+, z^{-}\right)$animals expressing Q40::YFP in muscle cells were viable but had increased embryonic arrest phenotype (Supplementary Figure $5 \mathrm{~h}$ ). Only the triple atg-3; $g s r-1(m+, z-) ;$ unc-52 failed to show an increased paralysis phenotype compared with the two control strains atg-3; unc-52 and $g s r-1(m+, z-)$; unc-52 (Fig. 5c). However, increasing the autophagy load by expressing the SQST-1::GFP autophagy substrate in unc$52 ; g s r-1\left(m+, z^{-}\right)$worms resulted in a much earlier paralysis onset compared with unc-52; $g s r-1\left(m+, z^{-}\right)$or unc-52; bpIs151 [Psqst-1::sqst-1::gfp] controls (Fig. 5d). Additional support to this hypothesis came from the generation of atg-3; gsr-1/qC1::rfp; bpIs151 worms, which produced very few $g s r-1(m+, z-)$ progeny that were very sick and arrested early during development . Furthermore, we also failed to isolate triples let-60; atg-3; bpIs151 or let-60; gsr$1\left(m+, z^{-}\right)$; bpIs 151 , reinforcing the notion that metastable LET-60 is also degraded by autophagy. Together, the fact that $g s r-1(m+, z-)$ mutants strongly enhance the deleterious phenotypes caused by aggregation-prone proteins in worms with compromised autophagy function (i.e., atg-3 mutations or by expression SQST-1::GFP protein) pinpoints a key role of GSR-1 in maintaining a healthy proteome.

Finally, to test whether the $g s r-1$ mutation impairs the autophagy flux, we employed a $C$. elegans strain that expresses a dual-fluorescent mCherry::GFP::LGG-1 fusion protein that monitors both APs (visualized as GFP and mCherry punctae) and autolysosomes (ALs; visualized only as mCherry puncta due to quenching of GFP due to the acidic environment of the AL) [48]. Using this reporter, we found that the autophagy flux is mildly affected by the $g s r-1$ mutation but became significantly impaired in unc-52; gsr-1 $\left(m+, z^{-}\right)$worms, as denoted by the increase of GFP/mCherry puncta and the decrease of mCherry only puncta in hypodermal seam cells (Fig. 5e). Moreover, we observed that expression of the mCherry::GFP::LGG-1 reporter in unc-52 mutants caused a reduction of worms length and that this phenotype was further enhanced in unc-52; $g s r-1(m+-z-)$ mutants (Supplementary Figure 5i). As a whole, these results identify GSR-1 as a novel positive regulator of autophagy.

\section{Glutathione reductase mutation and glutathione depletion increase protein aggregation in yeast and mammalian cell cultures}

To determine whether the protective role of the glutathione system on protein aggregation and associated phenotypes might be restricted to $C$. elegans or instead is a more universal, evolutionarily conserved mechanism, we first turned to a Saccharomyces cerevisiae model of polyQ toxicity [49]. Importantly, the mechanism by which glutathione reductase regulates polyQ aggregation appears to be maintained as yeast cells lacking glutathione reductase (carrying a $\Delta g l r l$ deletion) and expressing the aggregation-prone Q103-GFP fusion protein displayed a clear aggregation pattern, not detected in the control strain expressing a non-aggregating Q25-GFP fusion protein (Fig. 6a). Noteworthy, the aggregation phenotype of Q103-GFP expressing cells was much more evident in yeast that have reached the diauxic phase (Fig. 6a), when cells shift from a glucose fermentative to an ethanol respiration mitochondrial oxidative metabolism. 
a

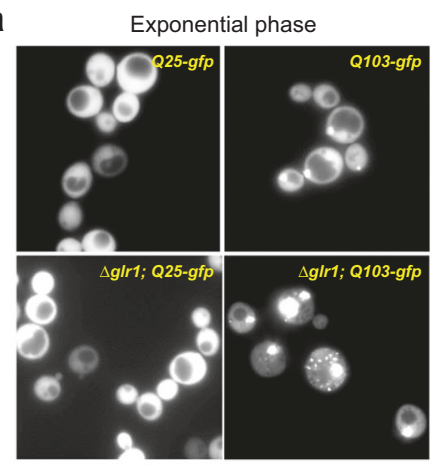

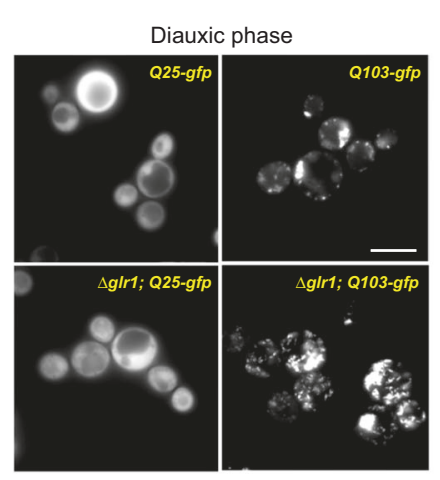

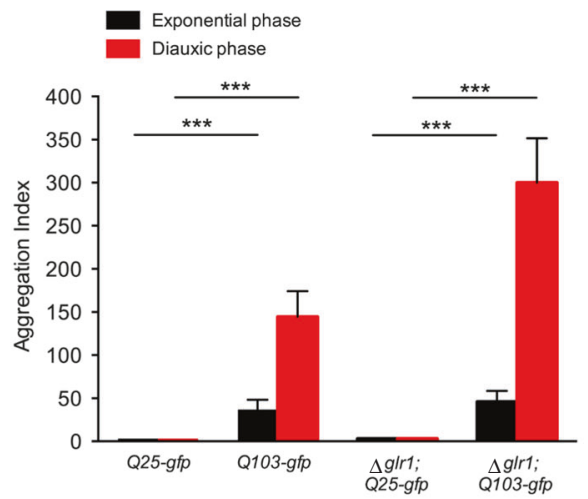

b
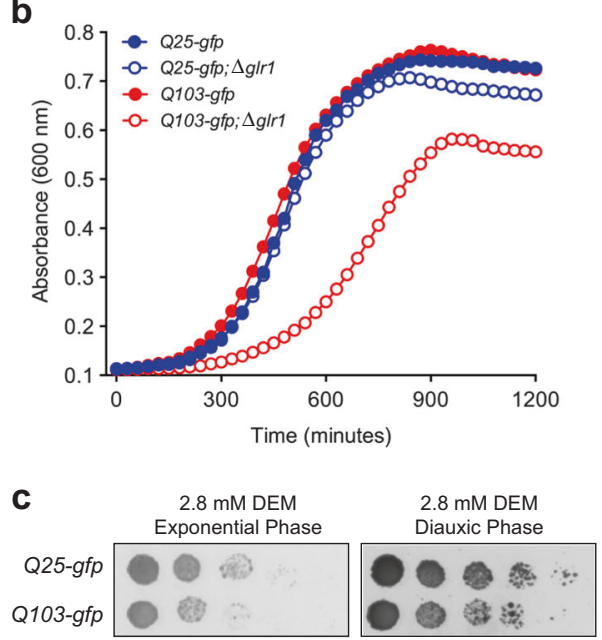

d

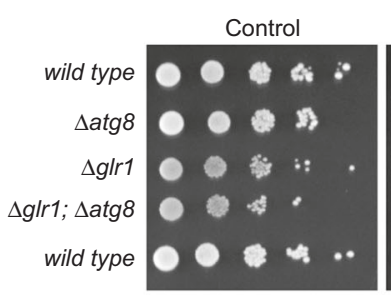

Q103-gfp

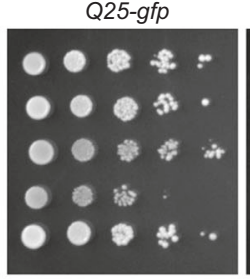

e

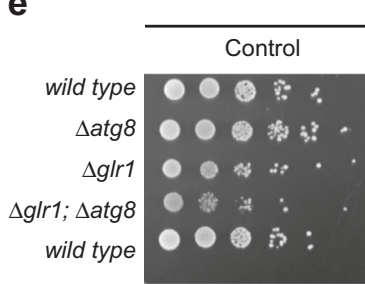

Q103-gfp

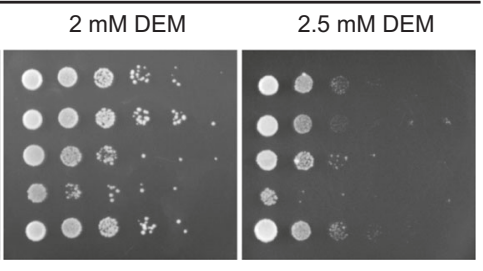

Fig. 6 The protective role of the glutathione system in proteostasis maintenance is conserved in yeast. WT and $\Delta g l r 1$ yeast strains expressing either 25Q-gfp or 103Q-gfp were grown in SC medium. a PolyQ-gfp fusion proteins were visualized by fluorescence microscopy and quantified (aggregation index) in cultures grown to exponential or diauxic phase as described in Materials and methods. The $\Delta g l r 1$ mutation increases the aggregation of 103Q-gfp but not 25Qgfp. Data are the mean \pm S.E.M. of three independent experiments with four biological replicates each. $* * * p<0.01$ by unpaired, two-tailed Student's $t$-test. Scale bar $6 \mu \mathrm{m}$. b Growth curves were measured in a microplate spectrophotometer for $20 \mathrm{~h}$ and only the $\Delta g l r 1$; $103 \mathrm{Q}$-gfp strain shows growth delay. The data are the averages of two independent experiments with three biological replicates each. c Viability of Q25-gfp and Q103-gfp yeast measured by plating serial dilutions (1:10) of cells grown to exponential or diauxic phase in SC plates without or with diethyl maleate (DEM; $2.8 \mathrm{mM}$ ). d, e Effect of $\Delta g l r l$ and/or $\Delta$ atg8 mutations on the viability of Q25-gfp and Q103-gfp yeast under (d) non-stressed conditions or (e) treated with DEM. Viability was measured by plating serial dilutions (1:10) of cells grown to diauxic phase prior to plating on SC plates
Indeed, protein aggregation of Q103-GFP was increased in $\Delta g l r l$ cells compared with a WT background only at diauxic phase, when oxidative stress generated by the metabolic shift occurred. In addition, Q103-gfp yeast had a significant growth delay in a $\Delta g l r l$ background and were more sensitive to DEM treatment than control Q25-GFP expressing cells (Fig. 6b, c), reminiscent of the larval developmental arrest phenotype described for Q40::YFP worms with downregulated $g s r-1$ levels (Supplementary Figure 1f) or exposed to DEM (Fig. 1b). To test whether autophagy is also involved in maintaining yeast proteostasis when redox homeostasis is challenged, we generated a double $\Delta$ glrl $\Delta a t g 8$ mutant in Q25-gfp and Q103-gfp backgrounds. atg8 encodes an ubiquitin-like protein, which is essential for AP formation [50]. As shown in Fig. 6d, single $\Delta g l r l$ or $\triangle a t g 8$ mutants grew similar to wild-type controls independently of expressing Q25-GFP or Q103-GFP proteins. However, a mild synthetic growth defect was detected in the double $\Delta g$ lrl 1 atg 8 in both $Q 25$-gfp and Q103-gfp backgrounds (Fig. 6d) and this synthetic interaction was further enhanced when cells were exposed to $2.5 \mathrm{mM}$ DEM (Fig. 6e). These data suggest that impairment of glutathione redox homeostasis negatively impacts on proteostasis in yeast cells expressing aggregation-prone proteins, involving autophagy as a mechanism to dismiss the increased load of protein aggregation.

Next, we addressed the protective effect of GSH homeostasis in a mammalian cell system. First, using a SH-SY5Y neuroblastoma cell line we evaluated whether, similar to worms, the subcellular localization dynamics of the HLH- 
30 mammalian orthologue TFEB is regulated by GSH. As previously described in other cell lines [40], while TFEB is mainly found in the cytosol when cells are cultivated in a complete nutritional medium, it strongly translocates into the nucleus upon starvation (Fig. 7a). However, when cells were starved in the presence of sublethal doses of DEM (Supplementary Figure 6), TFEB nuclear translocation was greatly hampered (Fig. 7a) indicating that TFEB dynamics is regulated by the glutathione system. We then asked whether GSH redox homeostasis is also required for proteostasis maintenance in mammals, and for this purpose we used a SH-SY5Y neuroblastoma cell line stably transfected with the human APP protein, which drives intracellular accumulation of human $A \beta$ [51]. Interestingly, when exposing these cells to sublethal doses of BSO and DEM (Supplementary Figure 6), we found that both treatments promoted an increase of $A \beta$ labeling in the cytoplasm of these cells (Fig. 7b). Moreover, the number of cells with $A \beta$ foci that colocalize with thioflavin, which labels amyloid fibrils and aggregates, was strongly induced upon BSO and DEM treatments (Fig. 7c). Given that SH-SY5Y cells overexpressing APP have enhanced autophagy [51], the increased $\mathrm{A} \beta$ aggregation observed in BSO and DEM treated cells is consistent with an impairment of autophagy.

Collectively, these data confirm that the deleterious effect of GSH and glutathione reductase deficiency on protein aggregation may be conserved from lower eukaryotes to mammals, impinging on the autophagy machinery.

\section{Discussion}

The maintenance of a functional proteome is essential for organismal survival and, among the molecular pathways that modulate proteostasis, redox regulation arises as a main regulatory mechanism allowing proteins to attain their native conformation and functionality. It has recently been proposed that the glutathione couple GSH/GSSG, rather than operating as a redox buffer would instead act as a surveillance relay to detect changes of the intracellular redox environment to tightly control the thioldisulfide balance of the cellular proteome [10]. In this study, we present a set of experiments that support this premise of the glutathione system being essential to preserve a healthy proteome, showing that genetic or pharmacological disruption of glutathione redox homeostasis enhances protein aggregation by a mechanism that impinges on the efficacy of autophagy. It is important to emphasize that our aim was to understand the relationship between redox homeostasis and proteotoxic stress, and not to disease per se, as it is still under debate whether insoluble aggregates or soluble oligomers are the toxic, pathological entity.

\section{Loss of glutathione redox homeostasis enhances the toxicity of protein aggregation}

Pharmacological inhibition of glutathione synthesis and recycling as well as glutathione depletion phenocopies the effect of the $g s r-1$ mutation in worms expressing aggregation-prone proteins. Surprisingly, treatment with DEM in $C$. elegans expressing A $\beta$, Q40::YFP and metastable UNC-52 proteins in muscle cells caused a fully penetrant lethality phenotype. DEM is an alkylating agent that has been traditionally used to deplete GSH but a recent study has shown that DEM depletes not only GSH but also protein thiols [52]. Hence, it is plausible that DEM causes an overload of oxidatively compromised proteins, possibly deemed for autophagic degradation, in addition to its direct effect on the GSH/GSSG ratio. This double impact may underlie the enhanced phenotypes observed in the worm proteostasis models exposed to DEM as compared with those carrying the gsr-1 mutation. Although it has been proposed that cytotoxicity can be genetically uncoupled from aggregation [53], we found a clear correlation of increased aggregation and DEM toxicity when aggregationprone proteins are expressed in muscle cells. However, this correlation was not so strong for proteins aggregating in neurons. We indeed found one case in which cytotoxicity inversely correlates with aggregation, namely in worms that express the human ATXN3::Q130::YFP protein in neurons and that are highly resistant to DEM treatment despite undergoing extensive aggregation, contrasting with worms that express non-aggregating ATXN3::Q75::YFP protein, which are sensitive to DEM (Figs. 2a, b). The resistance of ATXN3::Q130::YFP worms to the effects of DEM could be explained by an increase of exophers production, an extrusion mechanism mediated by very large extracellular vesicles containing damaged material such as aggregated proteins and that is enhanced when mitochondria are compromised [54], as is the case of $g s r-1$ mutants [20]. Interestingly, plasma membrane blebbing can also be observed in body wall muscle cells of transgenic worms expressing human $A \beta$, and these blebs contain $A \beta$ aggregates (Supplementary Figure 7a,b). Therefore, body wall muscle cells might also generate exophers under specific conditions and tissue-specific penetrance of the gsr-1 mutation or sensitivity to DEM treatment on exophers formation may account for the differential sensitivity of neuron versus muscle worm proteostasis models.

An unexpected phenotype identified in our experiments is the cell blebbing and explosion of $g s r-1\left(m-, z^{-}\right)$embryos that carry the rmIs133 [Punc-54::Q40::YFP] or $d v I s 2$ $[$ Punc-54:: human $A \beta]$ transgenes. To our knowledge, such striking phenotypes have not been previously described in C. elegans embryos. Cell blebs arise from transient disruption of the actomyosin cortex underneath the plasma 
a Complete Media
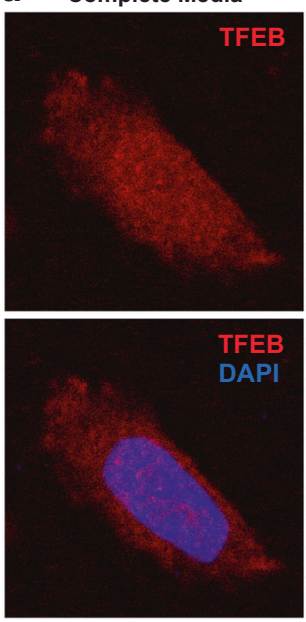

b
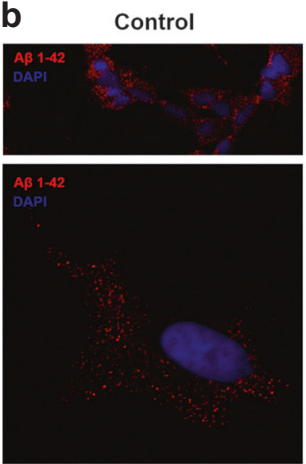

C
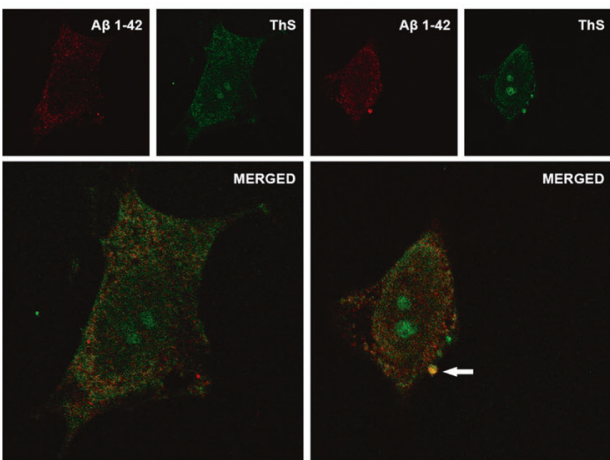

Fig. 7 The protective role of the glutathione system in proteostasis maintenance is conserved in mammals. a Confocal microscopy images of SH-SY5Y cells in complete medium or starved for $4 \mathrm{~h}$ in the absence or presence of DEM $(25 \mu \mathrm{M})$. Robust nuclear TFEB translocation is found after $4 \mathrm{~h}$ of starvation, which is prevented when cells were treated with $25 \mu \mathrm{M}$ diethyl maleate (DEM). TFEB is shown in red and DAPI staining for nuclei is shown in blue. Data are the mean \pm S.E.M. of three independent experiments ( $n=15$ images with at least 5 cells/image per assay and treatment). ${ }^{* * *} p<0.001$ by one-way analysis of variance (ANOVA) followed by Newman-Keuls' test. Scale bar $10 \mu \mathrm{m}$. b Confocal microscopy images of SH-SY5Y cells incubated for $18 \mathrm{~h}$ in the presence of BSO $(75 \mathrm{mM})$ and DEM $(25 \mu \mathrm{M})$.

membrane [28] and we were surprised to find that actin network distribution did not differ obviously from that of non-blebbing controls. In turn, we found that microtubules
Starvation $4 \mathrm{~h}+\mathrm{DEM} 25 \mu \mathrm{M}$
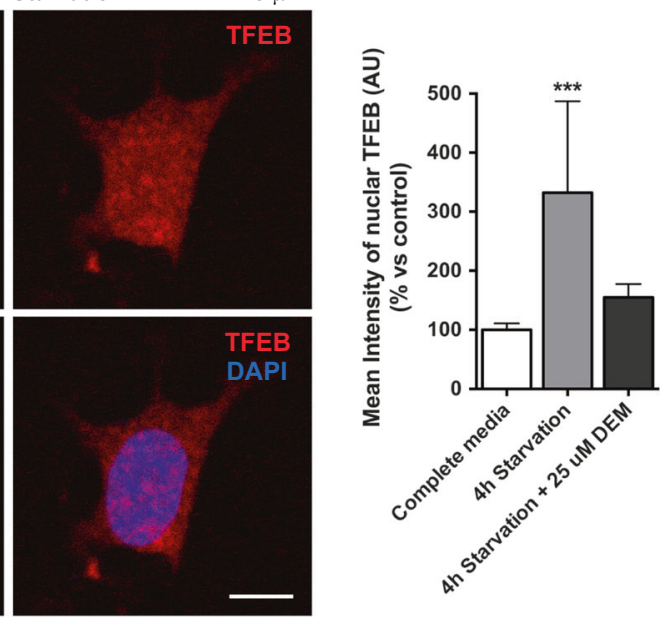

DEM $25 \mu \mathrm{M}$
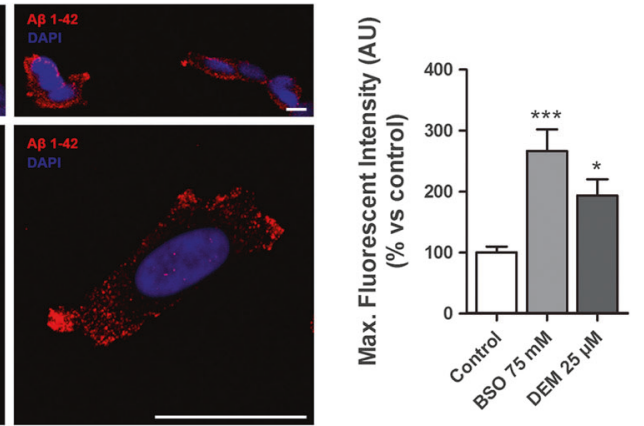

DEM $25 \mu \mathrm{M}$
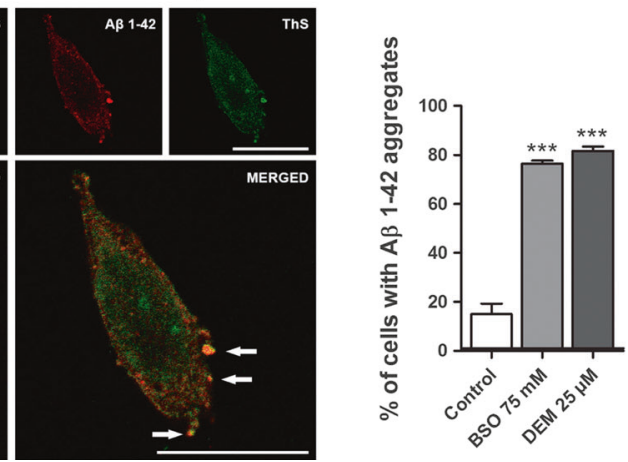

A clear increase of intracellular A $\beta 1-42$ staining is found in BSO and DEM-treated cells. A $\beta 1-42$ is shown in red and DAPI staining for nuclei is shown in blue. Data are the mean \pm S.E.M. of three independent experiments $(n=45$ images with at least 5 cells/image per assay and treatment). $* p<0.01, * * * p<0.001$ by one-way ANOVA followed by Newman-Keuls' test. Scale bar $30 \mu \mathrm{m}$. c The A $\beta 1-42$ aggregates in cells treated with BSO and DEM (in red), colocalize with amyloid fibrils labeled by Thioflavin S staining (in green). Colocalization is indicated by white arrows in the merged images. Data are the mean \pm S.E.M. of three independent experiments $(n \geq 50$ cells per assay and treatment). $* * * p<0.001$ by one-way ANOVA followed by Newman-Keuls' test. Scale bar $20 \mu \mathrm{m}$

were aberrantly distributed in the periphery of the $g s r-1(m-$, z-); rmIs133 [Punc-54::Q40::YFP] embryonic cells. A recent report has identified the worm calponin CHDP-1 
protein as promoter of cell protrusions in C. elegans. CHDP-1 is located at the cell cortex but, surprisingly, does not bind actin [55]. Instead CHDP-1 associates with the Rho small GTPase family member Rac1/CED-10, which, among other functions, mediates microtubule dynamics and lamellipodial protrusions [56-58]. Interestingly, we have found that ced-10(t1875) null mutant embryos also undergo cell blebbing, although these blebs are not as large and prominent as those observed in $g s r-1(m-, z-) ; r m I s 133$ embryos (Movie 7). Furthermore, we have recently reported that ced-10(n3246) mutants increase $\alpha$-synuclein::GFP aggregation in muscle cells and enhance $\alpha$-synuclein dependent dopaminergic neurodegeneration [59]. Similarly, ced-10 mutations also increase Q40::YFP aggregates in worm muscle cells (Supplementary Figure 7c), suggesting that CED-10 could mediate the protective effect of GSR-1 in these proteostasis models. How could GSR-1 control CED-10 function? One possibility is via glutathionylation (a post-translational modification whereby a glutathione moiety oxidizes a specific cysteine residue [60]), as mammalian Rac1 is glutathionylated at Cys18, a residue conserved in worm CED-10 protein, resulting in increased lamellipodia formation [61]. Given that glutathionylation is carried out by glutaredoxins, a class of small redox proteins that are reduced by GSH [62], it will be very interesting to explore whether glutaredoxins mediate the protective effect of GSR-1 in proteostasis maintenance.

\section{Role of cytoplasmic versus mitochondrial glutathione reductase in proteostasis}

Like the majority of eukaryotes, C. elegans uses the flavoenzyme glutathione reductase GSR-1 to recycle reduced glutathione from its oxidized form. In worms, $g s r-1$ is an essential gene that encodes two different GSR-1 isoforms, one located in the cytosol and the other in mitochondria. Although GSR-1 activity in the cytoplasm is absolutely required for embryonic development, consistent with glutathione synthesis occurring in this compartment, mitochondrial GSR-1 is dispensable under non-stressed conditions [20]. Surprisingly, $g s r-1\left(m+, z^{-}\right)$animals exhibit mitochondria-associated phenotypes such as mitochondrial fragmentation, induction of mitochondrial UPR, and lowered mitochondrial DNA content [20]. All the proteostasis models used in our study generate aggregated proteins in the cytosol, suggesting that the absence of GSR-1 activity in this compartment is responsible for the synthetic phenotypes observed in $g s r-1\left(m+, z^{-}\right)$mutants. However, we cannot rule out a possible role of the mitochondrial GSR-1 isoform as mitochondria have been shown to import and degrade aggregated proteins originally produced in the cytoplasm [63, 64]. Because $g s r-1(m+, z-)$ mutants display phenotypes associated with mitochondrial dysfunction, which might interfere with the import and degradation of the aggregated cytoplasmic proteins into this organelle, the implication of mitochondrial GSR-1 in proteostasis needs to be evaluated separately from the cytoplasmic isoform. Moreover, yeast Q103-GFP aggregates are more prominent in the diauxic phase, in which cells shift from a cytosolic fermentative to a mitochondrial respiratory metabolism, further supporting the possibility that mitochondrial glutathione reductase may have a role in protein aggregation.

\section{Glutathione-dependent regulation of autophagy}

Autophagy is an evolutionarily conserved degradation system of defective proteins and subcellular structures, and an early event in autophagy induction is the nuclear translocation and transactivation of the basic Helix-Loop-Helix transcription factor HLH-30/TFEB [39]. Oxidative stress is one of the various conditions that activate autophagy (reviewed in [65, 66]) and several components of the autophagy machinery are redox regulated by ROS-mediated mechanisms in different organisms [67-69]. Using a HyPer fluorescent biosensor [70], we found that $g s r-1\left(m+, z^{-}\right)$ worms have wild-type levels of $\mathrm{H}_{2} \mathrm{O}_{2}$ (A. Miranda-Vizuete, unpublished results), suggesting that they are not under oxidative stress and consistent with the lack of HLH-30: GFP nuclear translocation or increased SQST-1:GFP foci in these animals. Similarly, the $g s r-1$ mutation does not interfere with HLH-30 nuclear translocation upon heatshock, another ROS-producing treatment that induces autophagy [42, 71]. In turn, $g s r-1\left(m+, z^{-}\right)$mutants prevent HLH-30 nuclear translocation and degradation of the autophagy substrate p62/SQST-1 upon starvation, a condition that induces autophagy mainly through inhibition of the target of rapamycin (mTOR) pathway [72]. Together, these data suggest that the regulation of HLH-30 subcellular localization by GSR-1 is stimulus specific, likely mediated by mechanisms that do not involve ROS signaling.

We previously showed that $g s r-1$ mutants induce SKN-1 regulated genes of the glutathione pathway, like gcs- 1 and gst-4 [20]. Moreover, GSR-1 levels are strongly upregulated upon starvation by a mechanism dependent on the SKN-1 transcription factor [73]. Although SKN-1 may regulate mitophagy, a form of autophagy that specifically degrades defective mitochondria [74], it is not clear whether it can also regulate autophagy and protein aggregation in worms. Nonetheless, Nrf2 transcription factor, the SKN-1 mammalian orthologue, has been associated with autophagy and proteotoxicity $[75,76]$, thus supporting the possibility that SKN-1 could be a key player in the molecular mechanisms underlying GSR-1 function in these scenarios. Indeed, TFEB and Nrf2 transcription factors cooperate to stimulate autophagy in mammalian cells [77], suggesting that starvation-induced regulation of GSR-1 levels by these two 
transcription factors may be evolutionary conserved in worms.

Additional evidence of GSR-1 impinging on autophagy comes from the fact that $g s r-1\left(m+, z^{-}\right)$mutants further enhance the deleterious phenotypes caused by aggregationprone proteins in worms with compromised autophagy function either by atg-3 mutations or by overexpression of the SQST-1::GFP protein, which is specifically degraded by autophagy. This is nicely illustrated in unc-52; $g s r-1\left(m+, z^{-}\right)$; sqst-1::gfp worms, as they develop a much earlier onset of paralysis than the respective controls, probably by prioritizing SQST-1::GFP degradation and thus increasing the amount of aggregating UNC-52 that accelerates paralysis. Altogether, the synthetic phenotypes described in this study can be explained by a synergistic overload of misfolded proteins that cannot be degraded by autophagy, which ultimately surpasses a threshold over which cell function is severely affected eventually causing organismal death.

In summary, we found the glutathione system may be a major regulator of protein homeostasis as observed in $C$. elegans, yeast and mammalian cell models. Our data provide a new conceptual and experimental model to address the molecular mechanisms underlying redox regulation of proteostasis. Given the evolutionary conservation of this function, we postulate that strategies aimed to maintain glutathione redox homeostasis may have a therapeutic potential in diseases associated with protein aggregation, such as most prevalent neurodegenerative diseases.

\section{Materials and methods}

\section{C. elegans strains}

The standard methods used for culturing and maintenance of C. elegans were as described [78]. A list of all strains and plasmids used and generated in this study is provided in Supplementary Table 1. All VZ strains are 6x backcrossed with N2 wild-type. All transgenes used in this study are stably expressed from genomic integrated lines, except for strains carrying the $v z E x 169$ and $v z E x 170$ extrachromosomal arrays. Details on PCR, sequencing, or restriction enzyme genotyping of the different alleles, as well as plasmid constructs used in this work can be provided upon request. Unless otherwise noted, all experiments were performed on synchronized worms generated by allowing 10-15 gravid hermaphrodites to lay eggs during 2-3 h on seeded plates at $20^{\circ} \mathrm{C}$.

\section{S. cerevisiae strains}

A list of all yeast strains and plasmids used and generated in this study is provided in Supplementary Table 2. Yeast transformation was performed using a standard lithium acetate/polyethylene glycol method and all our experimentation used freshly transformed cells.

\section{DEM treatment}

Appropriate volumes from stock solutions of DEM (Sigma D97703, directly from the supplier, diluted in $0.5 \%$ DMSO final concentration on plates) were added to nematode growth medium (NGM) prior pouring the plates. Plates were freshly prepared to avoid degradation of the chemical with time. The developmental stages were scored in a SMZ645 Nikon stereoscope at specified days (depending on the growth temperature and genotype) after synchronized egg-lay.

\section{Quantification of polyQ C. elegans strains phenotypes}

polyQ::YFP aggregates quantification: For worms expressing the rmIs132 [Punc-54::Q35::yfp] and rmIs133 [Punc$54: \because Q 40 \because \because y f p]$ transgenes, muscle YFP aggregates were scored in the whole animal. Neuronal YFP aggregates in worms expressing the rmIs237 [Prgef-1::AT3v1-1::Q75:: $y f p]$ and rmIs263 [Prgef-1::AT3v1-1::Q130::yfp] transgenes were scored in the ventral nerve cord segment from the pharynx to the vulva. For worms expressing the $d r I s 20$ [Pvha-6::Q44::yfp] transgene, we scored the number of animals with 10 or more distinct YFP aggregates in the intestinal cells. All YFP aggregates quantifications were performed at $\times 40$ (muscle or intestinal aggregates) or $\times 100$ (neuronal aggregates) magnification in an Olympus BX61 fluorescence microscope equipped with a DP72 digital camera coupled to CellSens Software for image acquisition and analysis.

Mechanosensory response: The mechanosensory function in worms expressing the igIs245 [Pmec-3::htt57-19Q:: cfp] and igIs1 [Pmec-3::htt57-128Q::cfp] transgenes was assayed by the touch response assay [79]. Briefly, first day adult worms were gently touched on their tail with an eyelash mounted on a toothpick and their forward movement response was scored by 10 touches per worm assayed.

Time-lapse recording of blebbing and exploding embryo phenotypes: For the Differential interference contrast (DIC) recordings, embryos were prepared and mounted as described [80]. Gravid hermaphrodites were dissected, and two- to four-cell-stage embryos were mounted on $4 \%$ agar pads in water and sealed with Vaseline. 4D-microscopy (multifocal time-lapse microscopy) was carried out using a Leica DM6000 Microscope fitted with Nomarski optics. Recordings were made using a 100X/1.4 PL APO objective, and the temperature was kept constant at $25^{\circ} \mathrm{C}$. The microscope was controlled with the open-source software 
Micro-manager. Images from 30 focal planes ( $1 \mu \mathrm{m} / \mathrm{section})$ were taken every $30 \mathrm{~s}$ for up to $15 \mathrm{~h}$. Images and videos were processed with the Xnview and Quicktime software. For fluorescence recordings with the actin marker LifeAct:: mCherry, the microtubule marker GFP::tubulin and the chromatin marker mCherry::hisH2B, animals were grown at $16^{\circ} \mathrm{C}$ and shifted to $25^{\circ} \mathrm{C}$ approximately $24 \mathrm{~h}$ prior to observation. Gravid hermaphrodites were dissected and $g s r$ $1(m+, z+)$ and $g s r-1\left(m-, z^{-}\right)$embryos were carefully placed side by side on a $\sim 2 \%$ agarose pad in either $\mathrm{H}_{2} \mathrm{O}$ (Fig. $1 \mathrm{~g}$ ) or M9 (Fig. 1h). A coverslip was placed on top of the embryos and sealed with VALAP [81]. Confocal images were acquired on a Nikon A1R microscope through a 60x/1.4 PlanApo objective using a pinhole of 1.2 Airy Units $(39.7 \mu \mathrm{m})$; 3-5 focal planes separated by $2-3 \mu \mathrm{m}$ were recorded every $3-10 \mathrm{~min}$ for a total of $16 \mathrm{~h}$ at $22-24^{\circ} \mathrm{C}$. For mounting videos, a single focal plane was selected for each time point.

\section{Quantification of $\beta$-amyloid C. elegans strains phenotypes}

Paralysis: Worms expressing the $d v I s 2$ [Punc-54::human $A \beta]$ or $d v I s 14$ [Punc-54::human $A \beta$ ] transgenes (and respective controls) were grown at $16^{\circ} \mathrm{C}$ and paralysis scoring was initiated 4 days after egg-lay and determined daily, whereby paralyzed worms were removed from plates. A worm was scored as paralyzed if it did not respond to a gentle touch stimulus with a platinum wire.

Internal hatching: Animals carrying the $d v I s 14$ [Punc$54::$ human $A \beta$ ] transgene were grown at $16^{\circ} \mathrm{C}$ and the internal hatching phenotype was determined after adulthood by scoring their inability to extrude embryos, revealed by the absence of laid embryos in the area surrounding the vulva (as these animals are paralyzed). Images were captured using a SONY CCD camera (model DMK 31AU03) with the ImagingSource software (Imaging Control).

Developmental delay: Worms expressing the gnaIs 1 [Pmyo-2::yfp] and gnals2 [Punc-119::human A $\beta$; Pmyo-2:: $y f p]$ transgenes were grown at $20^{\circ} \mathrm{C}$ and their size was determined 3 days after egg-lay. Worms were imaged at $\times 10$ magnification in an Olympus BX61 fluorescence microscope equipped with a DP72 digital camera coupled to CellSens Software for image acquisition and analysis. ImageJ Software was used to quantify the length of the worms.

\section{Quantification of a-synuclein C. elegans strains phenotypes}

$\alpha$-synuclein::YFP aggregates quantification: For worms expressing the rmIs126 [Punc-54::yfp] and pkIs2386 [Punc$54:: \alpha$-synuclein::yfp] transgenes, the YFP aggregates were scored in the head muscle cells located within the segment encompassed by the two pharyngeal bulbs.

Dopaminergic neurodegeneration: Worms expressing

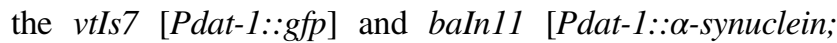
Pdat-1::gfp] transgenes were scored for neurodegeneration of the six anterior dopaminergic neurons (four CEP and two ADE dopaminergic neurons) according to previously described criteria [36]. Briefly, worms were scored as wildtype when all six dopaminergic (DA) neurons were present and their neuronal processes were intact while worms having DA cell body loss were scored as neurodegeneration.

YFP aggregates quantification and DA neuronal integrity were assessed at $\times 40$ magnification in an Olympus BX61 fluorescence microscope equipped with a DP72 digital camera coupled to CellSens Software for image acquisition and analysis.

Paralysis: Worms expressing the baIn 12 [Punc-54:: $\alpha$ synuclein::gfp] transgene (and respective controls) were grown at $20{ }^{\circ} \mathrm{C}$ and paralysis scoring was initiated 4 days after egg-lay and determined daily, whereby paralyzed worms were removed from plates. A worm was scored as paralyzed if it did not respond to a gentle touch stimulus with a platinum wire.

\section{Quantification of metastable protein C. elegans strains phenotypes}

unc-52(ts) paralysis: Worms carrying the unc-52 (e669su250) mutation were grown at $16{ }^{\circ} \mathrm{C}$ and paralysis was scored at the specified days after egg-lay, whereby paralyzed worms were removed from plates. A worm was scored as paralyzed if it did not respond to a gentle touch stimulus with a platinum wire.

let-60(ts) embryonic arrest: Worms carrying the let-60 (ga89) mutation were allowed to lay eggs at $16^{\circ} \mathrm{C}$ during $2-3 \mathrm{~h}$. Parents were removed, laid embryos counted, and then allowed to grow for 3 days at $16^{\circ} \mathrm{C}$. The embryonic arrest ratio was determined by dividing the number of developed larvae by the total number of laid embryos.

\section{HLH-30::GFP subcellular localization}

Worms expressing the sqIs17 [Phlh-30::hlh-30::GFP] transgene were scored at $\times 40$ magnification at the first day of adulthood for cytoplasmic versus nuclear GFP localization in an Olympus BX61 fluorescence microscope equipped with a DP72 digital camera coupled to CellSens Software for image acquisition and analysis. For the starvation assay, 1-day adult animals were incubated $1 \mathrm{~h}$ in non-seeded plates (supplemented with $100 \mu \mathrm{g} / \mathrm{ml}$ ampicillin to avoid bacterial growth) prior microscopy analysis. 


\section{SQST-1::GFP aggregates quantification}

Worms expressing the bpIs151 [Psqst-1::sqst-1::GFP] transgene were allowed to lay eggs at $20^{\circ} \mathrm{C}$ for $2-3 \mathrm{~h}$. Parents were removed and embryos were incubated during $6 \mathrm{~h}$. Embryos with GFP aggregates were scored at $\times 100$ magnification in the same microscopy setting as above. For the starvation assay, pregnant adults were incubated in M9 buffer at $20^{\circ} \mathrm{C}$ with gently shaking during $24 \mathrm{~h}$. Embryos accumulated in the uterus of the starved animals were freed by dissecting the parents with a needle and scored as described above.

\section{mCherry::GFP::LGG-1 punctae quantification}

Worm expressing the mCherry::GFP:LGG-1 transgene were grown at $16^{\circ} \mathrm{C}$ for 5 days after synchronized egglay. Autophagic vesicles were imaged in a Nikon A1R confocal microscope and z-stack images were acquired in hypodermal seam cells in $0.3 \mu \mathrm{m}$ slices at $63 \times$ amplification. Excitation/emission was set to $488 / 525 \mathrm{~nm}$ for GFP and 561/595 nm for mCherry. mCherry::GFP::LGG1 (mCherry/GFP or mCherry only) puncta were quantified in two well-defined hypodermal seam cells per animal in all $0.3 \mu \mathrm{m}$ slices per cell.

S. cerevisiae strains phenotypes and SH-SY5Y cells immunofluorescence protocols are detailed in Supplementary Methods section.

Acknowledgements We thank CGC (Caenorhabiditis Genetics Center) and NBRP (National BioResource Project), Richard Morimoto, Christian Pohl, Jan Gruber, Guy Caldwell, Randy Blakely, Hong Zhang, Enriq Herrero, Anne Bertolotti, and Susan Lindquist for providing strains and plasmids and Leticia Lemus for help with yeast viability assays. Cristina Ayuso García and the Live Cell Imaging Facility, Karolinska Institutet, Sweden (supported by grants from the Knut and Alice Wallenberg Foundation, the Swedish Research Council, the Centre for Innovative Medicine and the Jonasson Centre at RIT, Sweden) are acknowledged for technical assistance. The Spanish Ministry of Economy and Competitiveness supported EF-S and VG (BFU2016-78265-P), PA (BFU201679313-P and MDM-2016-0687), and AM-V (BFU2015-64408-P). AM-V was also supported by the Instituto de Salud Carlos III (PI11/ 00072) and RPV-M (CPII16/00004, PI14/00949 and PI17/00011). All projects were cofinanced by the Fondo Social Europeo (FEDER). AM-V is a member of the GENIE and EU-ROS Cost Actions of the European Union and RPV-M is a Marie Curie Fellow (CIG322034, EU).

Author contributions DG-G, JAM-L, and AM-V designed the study, performed most experiments using $C$. elegans, analyzed the data, and wrote the manuscript. BS-N and JC performed the embryo recordings, quantified the blebbing/exploding phenotypes, and contributed to the design of the study. FJN-G and FM-L carried out the initial candidate RNAi screen and generated several strains. CP-F, JG, and AC-M performed the mammalian cell experiments. CDL generated some strains and performed paralysis and DEM toxicity experiments. CN contributed essential reagents and analyzed the data. MDS and RPV-M generated $C$. elegans strains and carried out mechanosensory assays. EF-S, VG, RP, and EC contributed with the yeast experiments. PA performed the embryo confocal live imaging and immunostaining. All authors edited and revised the manuscript.

\section{Compliance with ethical standards}

Conflict of interest The authors declare that they have no conflict of interest.

Publisher's note: Springer Nature remains neutral with regard to jurisdictional claims in published maps and institutional affiliations.

\section{References}

1. Niforou K, Cheimonidou C, Trougakos IP. Molecular chaperones and proteostasis regulation during redox imbalance. Redox Biol. 2014;2:323-32.

2. Powers ET, Balch WE. Diversity in the origins of proteostasis networks--a driver for protein function in evolution. Nat Rev Mol Cell Biol. 2013;14:237-48.

3. Bayer TA. Proteinopathies, a core concept for understanding and ultimately treating degenerative disorders? Eur Neuropsychopharmacol. 2015;25:713-24.

4. Pievani M, Filippini N, van den Heuvel MP, Cappa SF, Frisoni GB. Brain connectivity in neurodegenerative diseases--from phenotype to proteinopathy. Nat Rev Neurol. 2014;10:620-33.

5. Go YM, Chandler JD, Jones DP. The cysteine proteome. Free Radic Biol Med. 2015;84:227-45.

6. Toledano MB, Delaunay-Moisan A, Outten CE, Igbaria A. Functions and cellular compartmentation of the thioredoxin and glutathione pathways in yeast. Antioxid Redox Signal. 2013;18:1699-711.

7. Lu J, Holmgren A. The thioredoxin superfamily in oxidative protein folding. Antioxid Redox Signal. 2014;21:457-70.

8. Poet GJ, Oka OB, van Lith M, Cao Z, Robinson PJ, Pringle MA, et al. Cytosolic thioredoxin reductase 1 is required for correct disulfide formation in the ER. EMBO J. 2017;36:693-702.

9. Ponsero AJ, Igbaria A, Darch MA, Miled S, Outten CE, Winther JR, et al. Endoplasmic reticulum transport of glutathione by Sec61 is regulated by Ero1 and Bip. Mol Cell. 2017;67:962-73.

10. Romero-Aristizabal C, Marks DS, Fontana W, Apfeld J. Regulated spatial organization and sensitivity of cytosolic protein oxidation in Caenorhabditis elegans. Nat Commun. 2014;5:5020.

11. Johnston AD, Ebert PR. The redox system in C. elegans, a phylogenetic approach. J Toxicol. 2012;2012:546915.

12. Miranda-Vizuete A, Veal EA. Caenorhabditis elegans as a model for understanding ROS function in physiology and disease. Redox Biol. 2017;11:708-14.

13. Feleciano DR, Arnsburg K, Kirstein J. Interplay between redox and protein homeostasis. Worm. 2016;5:e1170273.

14. Miranda-Vizuete A, Fierro Gonzalez JC, Gahmon G, Burghoorn J, Navas P, Swoboda P. Lifespan decrease in a Caenorhabditis elegans mutant lacking TRX-1, a thioredoxin expressed in ASJ sensory neurons. FEBS Lett. 2006;580:484-90.

15. Cacho-Valadez B, Munoz-Lobato F, Pedrajas JR, Cabello J, Fierro-Gonzalez JC, Navas P, et al. The characterization of the Caenorhabditis elegans mitochondrial thioredoxin system uncovers an unexpected protective role of thioredoxin reductase 2 in beta-amyloid peptide toxicity. Antioxid Redox Signal. 2012;16:1384-1400.

16. Stenvall J, Fierro-Gonzalez JC, Swoboda P, Saamarthy K, Cheng Q, Cacho-Valadez B, et al. Selenoprotein TRXR-1 and GSR-1 are 
essential for removal of old cuticle during molting in Caenorhabditis elegans. Proc Natl Acad Sci USA. 2011;108:1064-9.

17. Jimenez-Hidalgo M, Kurz CL, Pedrajas JR, Naranjo-Galindo FJ, Gonzalez-Barrios M, Cabello J, et al. Functional characterization of thioredoxin 3 (TRX-3), a Caenorhabditis elegans intestinespecific thioredoxin. Free Radic Biol Med. 2014;68:205-19.

18. Lei XG, Zhu JH, Cheng WH, Bao Y, Ho YS, Reddi AR, et al. Paradoxical roles of antioxidant enzymes: basic mechanisms and health implications. Physiol Rev. 2016;96:307-64.

19. Eriksson S, Prigge JR, Talago EA, Arner ES, Schmidt EE. Dietary methionine can sustain cytosolic redox homeostasis in the mouse liver. Nat Commun. 2015;6:6479.

20. Mora-Lorca JA, Saenz-Narciso B, Gaffney CJ, Naranjo-Galindo FJ, Pedrajas JR, Guerrero-Gomez D, et al. Glutathione reductase gsr-1 is an essential gene required for Caenorhabditis elegans early embryonic development. Free Radic Biol Med. 2016; 96:446-61.

21. Go YM, Jones DP. Redox compartmentalization in eukaryotic cells. Biochim Biophys Acta. 2008;1780:1273-90.

22. Kirstein J, Morito D, Kakihana T, Sugihara M, Minnen A, Hipp MS, et al. Proteotoxic stress and ageing triggers the loss of redox homeostasis across cellular compartments. EMBO J. 2015; 34:2334-49.

23. Edgley ML, Baillie DL, Riddle DL, Rose AM. Genetic balancers. WormBook 2006;6:1-32.

24. Link CD. Expression of human beta-amyloid peptide in transgenic Caenorhabditis elegans. Proc Natl Acad Sci USA. 1995;92:9368-72.

25. van Ham TJ, Thijssen KL, Breitling R, Hofstra RM, Plasterk RH, Nollen EA. C. elegans model identifies genetic modifiers of alphasynuclein inclusion formation during aging. PLoS Genet. 2008;4: e1000027.

26. Morley JF, Brignull HR, Weyers JJ, Morimoto RI. The threshold for polyglutamine-expansion protein aggregation and cellular toxicity is dynamic and influenced by aging in Caenorhabditis elegans. Proc Natl Acad Sci USA. 2002;99:10417-22.

27. Pollard TD, Cooper JA. Actin, a central player in cell shape and movement. Science. 2009;326:1208-12.

28. Fackler OT, Grosse R. Cell motility through plasma membrane blebbing. J Cell Biol. 2008;181:879-84.

29. Takesono A, Heasman SJ, Wojciak-Stothard B, Garg R, Ridley AJ. Microtubules regulate migratory polarity through Rho/ROCK signaling in T cells. PLoS ONE. 2010;5:e8774.

30. Sugiyama T, Pramanik MK, Yumura S. Microtubule-mediated inositol lipid signaling plays critical roles in regulation of blebbing. PLoS ONE. 2015;10:e0137032.

31. Teixeira-Castro A, Ailion M, Jalles A, Brignull HR, Vilaca JL, Dias N, et al. Neuron-specific proteotoxicity of mutant ataxin-3 in C. elegans: rescue by the DAF-16 and HSF-1 pathways. Hum Mol Genet. 2011;20:2996-3009.

32. Tourette C, Farina F, Vazquez-Manrique RP, Orfila AM, Voisin J, Hernandez S, et al. The Wnt receptor Ryk reduces neuronal and cell survival capacity by repressing FOXO activity during the early phases of mutant Huntingtin pathogenicity. PLoS Biol. 2014;12:e1001895.

33. Moronetti Mazzeo LE, Dersh D, Boccitto M, Kalb RG, Lamitina T. Stress and aging induce distinct polyQ protein aggregation states. Proc Natl Acad Sci USA. 2012;109:10587-92.

34. McColl G, Roberts BR, Pukala TL, Kenche VB, Roberts CM, Link CD, et al. Utility of an improved model of amyloid-beta (Abeta(1)(-)(4)(2)) toxicity in Caenorhabditis elegans for drug screening for Alzheimer's disease. Mol Neurodegener. 2012;7:57.

35. Fong S, Teo E, Ng LF, Chen CB, Lakshmanan LN, Tsoi SY, et al. Energy crisis precedes global metabolic failure in a novel Caenorhabditis elegans Alzheimer disease model. Sci Rep. 2016;6:33781.
36. Cao S, Gelwix CC, Caldwell KA, Caldwell GA. Torsin-mediated protection from cellular stress in the dopaminergic neurons of Caenorhabditis elegans. J Neurosci. 2005;25:3801-12.

37. Flames N, Hobert O. Gene regulatory logic of dopamine neuron differentiation. Nature. 2009;458:885-9.

38. Gidalevitz T, Ben-Zvi A, Ho KH, Brignull HR, Morimoto RI. Progressive disruption of cellular protein folding in models of polyglutamine diseases. Science. 2006;311:1471-4.

39. Lapierre LR, De Magalhaes Filho CD, McQuary PR, Chu CC, Visvikis O, Chang JT, et al. The TFEB orthologue HLH-30 regulates autophagy and modulates longevity in Caenorhabditis elegans. Nat Commun. 2013;4:2267.

40. Settembre C, Di Malta C, Polito VA, Garcia Arencibia M, Vetrini F, Erdin S, et al. TFEB links autophagy to lysosomal biogenesis. Science. 2011;332:1429-33.

41. O'Rourke EJ, Ruvkun G. MXL-3 and HLH-30 transcriptionally link lipolysis and autophagy to nutrient availability. Nat Cell Biol. 2013;15:668-76.

42. Kumsta C, Chang JT, Schmalz J, Hansen M. Hormetic heat stress and HSF-1 induce autophagy to improve survival and proteostasis in C. elegans. Nat Commun. 2017;8:14337.

43. Tian Y, Li Z, Hu W, Ren H, Tian E, Zhao Y, et al. C. elegans screen identifies autophagy genes specific to multicellular organisms. Cell. 2010;141:1042-55.

44. Jia K, Hart AC, Levine B. Autophagy genes protect against disease caused by polyglutamine expansion proteins in Caenorhabditis elegans. Autophagy. 2007;3:21-25.

45. Hamamichi S, Rivas RN, Knight AL, Cao S, Caldwell KA, Caldwell GA. Hypothesis-based RNAi screening identifies neuroprotective genes in a Parkinson's disease model. Proc Natl Acad Sci USA. 2008;105:728-33.

46. Florez-McClure ML, Hohsfield LA, Fonte G, Bealor MT, Link $\mathrm{CD}$. Decreased insulin-receptor signaling promotes the autophagic degradation of beta-amyloid peptide in C. elegans. Autophagy. 2007;3:569-80.

47. Dasgupta A, Zheng J, Bizzozero OA. Protein carbonylation and aggregation precede neuronal apoptosis induced by partial glutathione depletion. ASN Neuro. 2012;4:e00084.

48. Chang JT, Kumsta C, Hellman AB, Adams LM, Hansen M. Spatiotemporal regulation of autophagy during Caenorhabditis elegans aging. eLife 2017;6:e18459.

49. Dehay B, Bertolotti A. Critical role of the proline-rich region in Huntingtin for aggregation and cytotoxicity in yeast. J Biol Chem. 2006;281:35608-15.

50. Shpilka T, Weidberg H, Pietrokovski S, Elazar Z. Atg8: an autophagy-related ubiquitin-like protein family. Genome Biol. 2011;12:226.

51. Zheng L, Cedazo-Minguez A, Hallbeck M, Jerhammar F, Marcusson J, Terman A. Intracellular distribution of amyloid beta peptide and its relationship to the lysosomal system. Transl Neurodegener. 2012;1:19.

52. Urban N, Tsitsipatis D, Hausig F, Kreuzer K, Erler K, Stein V, et al. Non-linear impact of glutathione depletion on $C$. elegans life span and stress resistance. Redox Biol. 2017;11: $502-15$.

53. Gidalevitz T, Wang N, Deravaj T, Alexander-Floyd J, Morimoto RI. Natural genetic variation determines susceptibility to aggregation or toxicity in a C. elegans model for polyglutamine disease. BMC Biol. 2013;11:100.

54. Melentijevic I, Toth ML, Arnold ML, Guasp RJ, Harinath G, Nguyen KC, et al. C. elegans neurons jettison protein aggregates and mitochondria under neurotoxic stress. Nature. 2017;542: 367-71.

55. Guan L, Ma X, Zhang J, Liu JJ, Wang Y, Ding M. The calponin family member CHDP-1 interacts with Rac/CED-10 to promote cell protrusions. PLoS Genet. 2016;12:e1006163. 
56. Saenz-Narciso B, Gomez-Orte E, Zheleva A, Gastaca I, Cabello J. Control of developmental networks by Rac/Rho small GTPases: how cytoskeletal changes during embryogenesis are orchestrated. Bioessays. 2016;38:1246-54.

57. Wittmann T, Bokoch GM, Waterman-Storer CM. Regulation of leading edge microtubule and actin dynamics downstream of Rac1. J Cell Biol. 2003;161:845-51.

58. Waterman-Storer CM, Worthylake RA, Liu BP, Burridge K, Salmon ED. Microtubule growth activates Rac1 to promote lamellipodial protrusion in fibroblasts. Nat Cell Biol. 1999;1:45-50.

59. Kim H, Calatayud C, Guha S, Fernandez-Carasa I, Berkowitz L, Carballo-Carbajal I, et al. The small GTPase RAC1/CED-10 is essential in maintaining dopaminergic neuron function and survival against alpha-synuclein-induced toxicity. Mol Neurobiol. 2018;55:7533-52.

60. Mieyal JJ, Gallogly MM, Qanungo S, Sabens EA, Shelton MD. Molecular mechanisms and clinical implications of reversible protein S-glutathionylation. Antioxid Redox Signal. 2008;10:1941-88.

61. Hobbs GA, Mitchell LE, Arrington ME, Gunawardena HP, DeCristo MJ, Loeser RF, et al. Redox regulation of Rac1 by thiol oxidation. Free Radic Biol Med. 2015;79:237-50.

62. Shelton MD, Chock PB, Mieyal JJ. Glutaredoxin: role in reversible protein s-glutathionylation and regulation of redox signal transduction and protein translocation. Antioxid Redox Signal. 2005;7:348-66.

63. Zhou C, Slaughter BD, Unruh JR, Guo F, Yu Z, Mickey K, et al. Organelle-based aggregation and retention of damaged proteins in asymmetrically dividing cells. Cell. 2014;159:530-42.

64. Ruan L, Zhou C, Jin E, Kucharavy A, Zhang Y, Wen Z, et al. Cytosolic proteostasis through importing of misfolded proteins into mitochondria. Nature. 2017;543:443-6.

65. Navarro-Yepes J, Burns M, Anandhan A, Khalimonchuk O, del Razo LM, Quintanilla-Vega B, et al. Oxidative stress, redox signaling, and autophagy: cell death versus survival. Antioxid Redox Signal. 2014;21:66-85.

66. Filomeni G, De Zio D, Cecconi F. Oxidative stress and autophagy: the clash between damage and metabolic needs. Cell Death Differ. 2015;22:377-88.

67. Carroll B, Otten EG, Manni D, Stefanatos R, Menzies FM, Smith GR, et al. Oxidation of SQSTM1/p62 mediates the link between redox state and protein homeostasis. Nat Commun. 2018;9:256.

68. Perez-Perez ME, Zaffagnini $\mathrm{M}$, Marchand $\mathrm{CH}$, Crespo JL, Lemaire SD. The yeast autophagy protease Atg4 is regulated by thioredoxin. Autophagy. 2014;10:1953-64.
69. Frudd K, Burgoyne T, Burgoyne JR. Oxidation of Atg3 and Atg7 mediates inhibition of autophagy. Nat Commun. 2018;9:95.

70. Back P, De Vos WH, Depuydt GG, Matthijssens F, Vanfleteren JR, Braeckman BP. Exploring real-time in vivo redox biology of developing and aging Caenorhabditis elegans. Free Radic Biol Med. 2012;52:850-9.

71. Banerjee Mustafi S, Chakraborty PK, Dey RS, Raha S. Heat stress upregulates chaperone heat shock protein 70 and antioxidant manganese superoxide dismutase through reactive oxygen species (ROS), p38MAPK, and Akt. Cell Stress Chaperon-. 2009;14:579-89.

72. Dunlop EA, Tee AR. mTOR and autophagy: a dynamic relationship governed by nutrients and energy. Semin Cell Dev Biol. 2014;36:121-9.

73. Luersen K, Stegehake D, Daniel J, Drescher M, Ajonina I, Ajonina $\mathrm{C}$, et al. The glutathione reductase GSR-1 determines stress tolerance and longevity in Caenorhabditis elegans. PLoS ONE. 2013;8:e60731.

74. Palikaras K, Lionaki E, Tavernarakis N. Coordination of mitophagy and mitochondrial biogenesis during ageing in C. elegans. Nature. 2015;521:525-8.

75. Dodson M, Redmann M, Rajasekaran NS, Darley-Usmar V, Zhang J. KEAP1-NRF2 signalling and autophagy in protection against oxidative and reductive proteotoxicity. Biochem $\mathrm{J}$. 2015;469:347-55.

76. Jiang T, Harder B, Rojo de la Vega M, Wong PK, Chapman E, Zhang DD. p62 links autophagy and Nrf2 signaling. Free Radic Biol Med. 2015;88:199-204.

77. Kim S, Choi KJ, Cho SJ, Yun SM, Jeon JP, Koh YH, et al. Fisetin stimulates autophagic degradation of phosphorylated tau via the activation of TFEB and Nrf2 transcription factors. Sci Rep. 2016;6:24933.

78. Stiernagle T. Maintenance of C. elegans. WormBook 2006;11:1-11.

79. Parker JA, Connolly JB, Wellington C, Hayden M, Dausset J, Neri C. Expanded polyglutamines in Caenorhabditis elegans cause axonal abnormalities and severe dysfunction of PLM mechanosensory neurons without cell death. Proc Natl Acad Sci USA. 2001;98:13318-23.

80. Nieto C, Almendinger J, Gysi S, Gomez-Orte E, Kaech A, Hengartner MO, et al. ccz-1 mediates the digestion of apoptotic corpses in C. elegans. J Cell Sci. 2010;123:2001-7.

81. Askjaer P, Galy V, Meister P. Modern tools to study nuclear pore complexes and nucleocytoplasmic transport in Caenorhabditis elegans. Methods Cell Biol. 2014;122:277-310.

\section{Affiliations}

\section{David Guerrero-Gómez ${ }^{1}$ José Antonio Mora-Lorca ${ }^{1,2} \cdot$ Beatriz Sáenz-Narciso ${ }^{3}$ - Francisco José Naranjo-Galindo ${ }^{1}$. Fernando Muñoz-Lobato ${ }^{1}$ - Cristina Parrado-Fernández ${ }^{4}$ - Julen Goikolea ${ }^{4}$ - Ángel Cedazo-Minguez ${ }^{4}$. Christopher D. Link ${ }^{5}$. Christian Neri ${ }^{6} \cdot$ María Dolores Sequedo ${ }^{7,8} \cdot$ Rafael P. Vázquez-Manrique $^{7,8}$. Elena Fernández-Suárez ${ }^{9} \cdot$ Veit Goder $^{9} \cdot$ Roser Pané $^{10} \cdot$ Elisa Cabiscol $^{10} \cdot$ Peter Askjaer $^{11}$ - Juan Cabello ${ }^{3}$. Antonio Miranda-Vizuete $\mathbb{1}^{1}$}

1 Redox Homeostasis Group, Instituto de Biomedicina de Sevilla (IBIS), Hospital Universitario Virgen del Rocío/CSIC/Universidad de Sevilla, 41013 Sevilla, Spain

2 Departamento de Farmacología, Facultad de Farmacia, Universidad de Sevilla, 41012 Sevilla, Spain

3 CIBIR (Center for Biomedical Research of La Rioja), 26006 Logroño, Spain
4 Karolinska Institutet, Department of Neurobiology, Care Sciences and Society, Center for Alzheimer Research, Division of Neurogeriatrics, Stockholm SE-14186, Sweden

5 Department of Integrative Physiology, Institute for Behavioral Genetics, University of Colorado at Boulder, Boulder, CO 80309, USA

6 Sorbonnes Université, Centre National de la Recherche 
Scientifique, Research Unit Biology of Adaptation and Aging (B2A), Team Compensation in Neurodegenerative and Aging (Brain-C), F-75252 Paris, France

7 Research Group in Molecular, Cellular and Genomic Biomedicine, Health Research Institute-La Fe, 46026 Valencia, Spain

8 Centro de Investigación Biomédica en Red de Enfermedades Raras (CIBERER), Valencia, Spain
9 Departamento de Genética, Facultad de Biología, Universidad de Sevilla, 41012 Sevilla, Spain

10 Departament de Ciències Mèdiques Bàsiques, IRB Lleida, Universitat de Lleida, Av. Rovira Roure, 80, 25198 Lleida, Spain

11 Andalusian Center for Developmental Biology (CABD), CSIC/ JA/Universidad Pablo de Olavide, 41013 Seville, Spain 